\title{
RIGHT UNIMODAL AND BIMODAL SINGULARITIES IN POSITIVE CHARACTERISTIC
}

\author{
NGUYEN HONG DUC
}

\begin{abstract}
The problem of classification of real and complex singularities was initiated by Arnol'd in the sixties who classified simple, unimodal and bimodal singularities w.r.t. right equivalence. The classification of simple singularities positive characteristic was achieved by Greuel and the author in 2014. In the present paper we classify right unimodal and bimodal singularities in positive characteristic by giving explicit normal forms. It is surprising that in positive characteristic, there are no infinite series of unimodal and bimodal singularities. Moreover, the Milnor number of simple, unimodal and bimodal singularity satisfies $\mu(f) \leq 4 p$. As an application we prove that, for singularities of right modality at most 2 , the $\mu$-constant stratum is smooth and its dimension is equal to the right modality.
\end{abstract}

\section{INTRODUCTION}

We classify hypersurface singularities $f \in K\left[\left[x_{1}, \ldots, x_{n}\right]\right]$ which are unimodal and bimodal w.r.t. right equivalence, where $K$ is an algebraically closed field of positive characteristic. That is, the singularities have modality 1 resp. 2 up to the change of coordinates (or right equivalence, see Section 2.1). The notion of modality was introduced by Arnol'd in the seventies into the singularity theory for real and complex singularities. He classified simple, unimodal and bimodal hypersurface singularities w.r.t. right equivalence ([2, [3, [5]). He showed that the simple singularities are the $A D E$-singularities, i.e. the two infinite series $A_{k}, k \geq 1, D_{k}, k \geq 4$, and the three exceptional singularities $E_{6}, E_{7}, E_{8}$. The unimodal singularities include three families of parabolic singularities, a three-index family of hyperbolic singularities and 14 exceptional singularities. There are 8 infinite series and 14 exceptional bimodal singularities. The unimodal and bimodal singularities are also classified by degenerations of elliptic curves ([16]), which were studied by Kodaira. In positive characteristic, the right simple singularities were recently classified by Greuel and the author in [14.

The main result of the present paper is the classification of right unimodal and bimodal singularities in positive characteristic with tables of normal forms. Our lists of normal forms for unimodal and bimodal singularities are given in $\$ 3$. A surprising fact of our classification is that in positive characteristic, there are no infinite series of unimodal and bimodal singularities. Moreover, the Milnor number of simple, unimodal and bimodal singularity satisfies $\mu(f) \leq 4 p$ (Corollary 3.7). Another surprising fact is that, some $A$ - or $D$-singularities occurs in the lists of unimodal and bimodal singularities. On the other hand, if the characteristic is 2 or 3, then all simple, unimodal and bimodal singularities must be of type $A, D$ or $E$. The adjacency diagrams of simple, unimodal and bimodal singularities are also determined (Theorem 3.5. As an application of the classification, we show that the $\mu$-constant stratum of simple, unimodal and bimodal singularities is smooth and its dimension coincides with the right modality (Corollary $3.8,3.9$ ).

We organize our proof in the form of a singularity determinator ( $\S$ (4), finding for every given singularities its place in the list of $\S 3$. This method was used by Arnol'd in [6] to study local normal

Date: August 11, 2017.

2010 Mathematics Subject Classification. Primary 14B05; Secondary 14J10.

${ }^{\dagger}$ This research project was partially supported by Vietnam National Foundation for Science and Technology Development (NAFOSTED) grant 101.04-2014.23, the Oberwolfach Leibniz Fellows programme of the Mathematisches Forschungsinstitut Oberwolfach (Germany), the ERCEA Consolidator Grant 615655 NMST and also by the Basque Government through the BERC 2014-2017 program and by Spanish Ministry of Economy and Competitiveness MINECO: BCAM Severo Ochoa excellence accreditation SEV-2013-0323. 
forms of complex functions. He formed a singularity determinator to recover the classification of simple, unimodal and bimodal singularities, to obtain classifications of all the singularities with Milnor number $\mu \leq 16$, all the singularities of corank 2 with nonzero 4 -jet, all the singularities of corank 3 with a 3-jet, which determine an irreducible cubic, and some other singularities. However, in positive characteristic, we obtain only the classification of simple, unimodal and bimodal singularities; the other classifications are still unknown. It seems that the classification problems are very different in positive characteristic even for the singularities of corank 1 ([22]). The main difficulty is to find for each series $A_{k}, D_{k}, J_{2, q}, \ldots$ in $\S 3$ the exact bound of its index. This also shows that, there are only finitely many series of simple, unimodal and bimodal singularities.

Note that, for contact equivalence and for $K=\mathbb{C}$, it was proved by Giusti in [1] that $A D E$ singularities are contact simple. The classification of contact unimodal singularities was achieved by Wall in [24. Greuel and Kröning showed in [12] that the contact simple singularities over a field of positive characteristic are again exactly the $A D E$-singularities or the rational double points of Artin's list [7.

\section{Modality}

Modality was introduced by Arnol'd in connection with the classification of singularities of functions under right equivalence. It has been generalized to arbitrary actions of algebraic groups by Vinberg [18]. Wall [20] described two possible generalizations for use in other classification problems in singularity theory. Both generalizations are developed in detail by Greuel and the author ([14]) for any characteristic and it was proved that they coincide.

2.1. Right modality. Consider an action of algebraic group $G$ on a variety $X$ (over a given algebraically closed field $K)$ and a Rosenlicht stratification $\left\{\left(X_{i}, p_{i}\right), i=1, \ldots, s\right\}$ of $X$ w.r.t. $G$. That is, a stratification $X=\cup_{i=1}^{s} X_{i}$, where the stratum $X_{i}$ is a locally closed $G$-invariant subvariety of $X$ such that the projection $p_{i}: X_{i} \rightarrow X_{i} / G$ is a geometric quotient. For each open subset $U \subset X$ the modality of $U, G-\bmod (U)$, is the maximal dimension of the images of $U \cap X_{i}$ in $X_{i} / G$. The modality $G-\bmod (x)$ of a point $x \in X$ is the minimum of $G$-mod $(U)$ over all open neighbourhoods $U$ of $x$.

Let $K[[\mathbf{x}]]=K\left[\left[x_{1}, \ldots, x_{n}\right]\right]$ the formal power series ring and let the right group, $\mathcal{R}:=\operatorname{Aut}(K[[\mathbf{x}]])$, act on $K[[\mathbf{x}]]$ by $(\Phi, f) \mapsto \Phi(f)$. Two elements $f, g \in K[[\mathbf{x}]]$ are called right equivalent, $f \sim_{r} g$, if they belong to the same $\mathcal{R}$-orbit, or equivalently, there exists a coordinate change $\Phi \in A u t(K[[\mathbf{x}]])$ such that $g=\Phi(f)$.

Recall that for $f \in \mathfrak{m} \subset K[[\mathbf{x}]], \mu(f):=\operatorname{dim} K[[\mathbf{x}]] / j(f), j(f)=\left\langle f_{x_{1}}, \ldots, f_{x_{n}}\right\rangle$, denotes the Milnor number of $f$ and that $f$ is isolated if $\mu(f)<\infty$. The $k$-jet of $f, j^{k}(f)$, is the image of $f$ in the jet space $J_{k}:=\mathfrak{m} / \mathfrak{m}^{k+1}$. We call $f$ to be right $k$-determined if each singularity having the same $k$-jet with $f$, is right equivalent to $f$. A number $k$ is called right sufficiently large for $f$, if there exists a neighbourhood $U$ of the $j^{k} f$ in $J_{k}$ such that every $g \in K[[\mathbf{x}]]$ with $j^{k} g \in U$ is right $k$-determined. The right modality of $f, \mathcal{R}$ - $\bmod (f)$, is defined to be the $\mathcal{R}_{k}$-modality of $j^{k} f$ in $J_{k}$ with $k$ right sufficiently large for $f$, where $\mathcal{R}_{k}$ the $k$-jet of $\mathcal{R}$. A singularity $f \in K[[\mathbf{x}]]$ is called (right) simple, uni-modal, bi-modal and $r$-modal if its (right) modality is equal to 0,1,2 and $r$ respectively. These notions are independent of the right sufficiently large $k$ ([14, Def. 2.3).

The second description is in relation with versal or complete deformation. Let $T$ be an affine variety with its algebra of global section $\mathcal{O}(T)$. Then a family $f_{t}(\mathbf{x}):=F(\mathbf{x}, t) \in \mathcal{O}(T)[[\mathbf{x}]]$ is called an unfolding (deformation with trivial section) of $f$ over a pointed space $T, t_{0}$ if $F\left(\mathbf{x}, t_{0}\right)=f$ and $f_{t} \in \mathfrak{m}$ for all $t \in T$. For a basis $\left\{\mathbf{x}^{\alpha_{1}}, \ldots, \mathbf{x}^{\alpha_{N}}\right\}$ of $\mathfrak{m} / \mathfrak{m} \cdot j(f)$, the semiuniversal unfolding is represented by $F(\mathbf{x}, \lambda):=f(\mathbf{x})+\sum_{i=1}^{N} \lambda_{i} \mathbf{x}^{\alpha_{i}}$ with $\lambda=\left(\lambda_{1}, \ldots, \lambda_{N}\right)$ the coordinate of $\lambda \in \mathbb{A}^{N}$. Note that from the exact sequence

$$
0 \rightarrow j(f) / \mathfrak{m} \cdot j(f) \rightarrow \mathfrak{m} / \mathfrak{m} \cdot j(f) \rightarrow \mathfrak{m} / j(f) \rightarrow 0
$$

we get $N=\mu+n-1$. Since $\mathfrak{m} \cdot j(f)$ is the tangent space of the orbit of the right group $\mathcal{R}$ at $f([9])$, $N$ is the codimension of the orbit in $\mathfrak{m}$.

An unfolding $F(\mathbf{x}, t)$ over $T, t_{0}$ is called right complete if any unfolding $H(\mathbf{x}, s)$ over $S, s_{0}$ is isomorphic to a pullback of $F$ after passing to some étale neighbourhood of $S, s_{0}$, see [14]. An important 
property of the complete unfoldings is that they are sufficient to determine the modality, i.e. if $F$ is a complete unfolding of $f$, then modality of $f$ w.r.t. $F$ ([14, Def. 2.5]) equals to modality of $f$, see [14, Prop. 2.12(ii)]. The semiuniversal unfolding of an isolated hypersurface singularity is right complete (see [15] for the analytic case and [14] for the general case). Consequently, we may define modality of $f$ as follows: "Let $f_{\lambda}$ be the semiuniversal unfolding of $f$ over $\mathbb{A}^{N}, 0$. If the set of singularities $f_{\lambda} \in K[[\mathbf{x}]]$ ( $\lambda$ in some Zariski neighbourhood of $0 \in \mathbb{A}^{N}$ ) falls into finitely many families of right classes, each depending on $r$ parameters (at most) then $f$ is right (resp. contact) $r$-modal (at most)."

Remark 2.1. (1) For convergent power series over the complex numbers it does not make any difference wheter we consider the semiuniversal deformation (without section) given by the Milnor algebra $\mathbb{C}\left\{x_{1}, \ldots, x_{n}\right\} / j(f)$ or the semiuniversal deformation with section given by $\mathfrak{m} / \mathfrak{m} \cdot j(f)$. However, in positive characteristic we have to consider the latter (cf. 14] and 2.2).

(2) The difference between the classical versal and our complete deformation is twofold. First, we consider deformations over algebraic varieties and not just of the spectrum of a complete local ring (as for versal deformations). Second, we do not require the lifting property for induced deformations over small extensions (cf. [13, Ch.2]).

2.2. Proper modality. In [10] Gabrielov showed in the complex analytic case that the right modality is equal to the dimension of the $\mu$-constant stratum in a semi-universal deformation of $f$. This is not true in positive characteristic since $f=x^{2}+y^{4} \in A_{3} \subset K[[x, y]]$ with $\operatorname{char}(K)=3$, is unimodal, but the dimension of the stratum $\mu=3$ into the semiuniversal deformation $f+a_{0}+a_{1} y+a_{2} y^{2}$, is equal to 0 . In positive characteristic we need to consider deformations with section. Let $f_{\lambda}(\mathbf{x}):=F(\mathbf{x}, \lambda)$ be the semiuniversal unfolding of $f$ with trivial section over affine variety $\mathbb{A}^{N}, 0$ with $N=\mu+n-1$ as above. We define the proper modality of $f$, denoted by $\operatorname{pmod}(f)$, to be the dimension at 0 of the $\mu$-constant stratum in $\mathbb{A}^{N}$ :

$$
\Delta_{\mu}:=\left\{\lambda \in \mathbb{A}^{N} \mid \mu\left(f_{\lambda}\right)=\mu\right\} .
$$

Conjecture 2.2. $\operatorname{pmod}(f)=\operatorname{rmod}(f)$.

See Corollary 3.9 for a partial result of the conjecture. Namely, if $\operatorname{rmod}(f) \leq 2$ then $\operatorname{pmod}(f)=$ $\operatorname{rmod}(f)$.

\section{Right Unimodal, BIMOdAl Singularities AND ADJACENCY DiAGRAMS}

In this section we present the result of our classification by explicit lists of normal forms, the adjacency diagrams of simple, unimodal and bimodal singularities, and their applications. Recall that a normal form is a modular family $F(\mathbf{x}, t) \in \mathcal{O}(T)[[\mathbf{x}]]$, i.e. for each $t \in T$ there are only finitely many $t^{\prime} \in T$ such that $f_{t^{\prime}} \sim_{r} f_{t}$. Notice that, if $F(\mathbf{x}, t)$ is a normal form, then the right modality of $F(\mathbf{x}, t)$ for all $t \in T$, is not less than the dimension of $T$.

\subsection{Right unimodal singularities.}

Theorem 3.1. Let $p=\operatorname{char}(K)>2$. A hypersurface singularity $f \in \mathfrak{m}^{2}$ is right unimodal if and only if it is right equivalent to one of the following forms:

I. $n=1(f \in K[[x]])$. The classification is given in Table 1 .

II. $n=2(f \in K[[x, y]])$. The classification is given in Table 2.

III. $n=3(f \in K[[x, y, z]])$. The classification is given in Table 3 .

IV. $n>3$. The classification is given in Table 4.

Theorem 3.2. Let $p=\operatorname{char}(K)=2$. A hypersurface singularity $f \in \mathfrak{m}^{2}$ is right unimodal if and only if $n$ is odd and $f$ is right equivalent to one of singularities in the Table 5 . 


\begin{tabular}{clll}
\hline Name & Normal form & Conditions & $\mu$ \\
\hline $\mathrm{A}_{k}$ & $x^{p}+a x^{k+1}$ & $p \leq k \leq 2 p-2$ & $k$ \\
\hline
\end{tabular}

TABLE 1 . Unimodal singularities $(p>2, n=1)$

\begin{tabular}{clll}
\hline Name & Normal form & Conditions & $\mu$ \\
\hline $\mathrm{A}_{k}$ & $x^{2}+a y^{p}+y^{k+1}$ & $p \leq k \leq 2 p-2$ & $k$ \\
$\mathrm{D}_{p}$ & $x^{2} y+y^{p-1}$ & $3<p$ & $p$ \\
$\mathrm{D}_{k}$ & $x^{2} y+a y^{p}+y^{k-1}$ & $3 \leq p<k-1 \leq 2 p-2$ & $k$ \\
$\mathrm{E}_{12}$ & $x^{3}+y^{7}+a x y^{5}$ & $7<p$ & 12 \\
$\mathrm{E}_{13}$ & $x^{3}+x y^{5}+a y^{8}$ & $7<p$ & 13 \\
$\mathrm{E}_{14}$ & $x^{3}+y^{8}+a x y^{6}$ & $7<p$ & 14 \\
$\mathrm{~J}_{10}=\mathrm{J}_{2,0}=\mathrm{T}_{2,3,6}$ & $x^{3}+y^{6}+a x^{2} y^{2}$ & $5<p$ & 10 \\
$\mathrm{~J}_{2, q}$ & $x^{3}+a x^{2} y^{2}+y^{6+q}$ & $6<6+q<p$ & $q+10$ \\
$\mathrm{~W}_{12}$ & $x^{4}+y^{5}+a x^{2} y^{3}$ & $p>5$ & 12 \\
$\mathrm{~W}_{13}$ & $x^{4}+x y^{4}+a y^{6}$ & $p>5$ & 9 \\
$\mathrm{X}_{9}=\mathrm{X}_{1,0}=\mathrm{T}_{2,4,4}$ & $x^{4}+y^{4}+a x^{2} y^{2}$ & $3<p$ & $9+9$ \\
$\mathrm{X}_{1, q}=\mathrm{T}_{2,4,4+q}$ & $x^{4}+x^{2} y^{2}+a y^{4+q}$ & $4<4+q<p$ & $9+r+s$ \\
$\mathrm{Y}_{r, s}=\mathrm{T}_{2,4+r, 4+s}$ & $x^{4+r}+a x^{2} y^{2}+y^{4+s}$ & $4<4+r \leq 4+s<p$ & 11 \\
$\mathrm{Z}_{11}$ & $x^{3} y+y^{5}+a x y^{4}$ & $5<p$ & 12 \\
$\mathrm{Z}_{12}$ & $x^{3} y+x y^{4}+a x^{2} y^{3}$ & $5<p$ & 13 \\
$\mathrm{Z}_{13}$ & $x^{3} y+y^{6}+a x y^{5}$ & $5<p$ &
\end{tabular}

TABLE 2. Unimodal singularities $(p>2, n=2)$

\begin{tabular}{|c|c|c|c|}
\hline Name & Normal form & Conditions & $\mu$ \\
\hline & $g(x, y)+z^{2}$ & $g$ one of the series in Table 2 & $\mu(g)$ \\
\hline $\mathrm{P}_{8}=\mathrm{T}_{3,3,3}$ & $x^{3}+y^{3}+z^{3}+a x y z$ & $3<p$ & 8 \\
\hline $\mathrm{Q}_{10}$ & $x^{3}+y^{4}+y z^{2}+a x y^{3}$ & $3<p$ & 10 \\
\hline $\mathrm{Q}_{11}$ & $x^{3}+y z^{2}+x z^{3}+a z^{5}$ & $3<p$ & 11 \\
\hline $\mathrm{Q}_{12}$ & $x^{3}+y^{5}+y z^{2}+a x y^{4}$ & $5<p$ & 12 \\
\hline $\mathrm{S}_{11}$ & $x^{4}+y^{2} z+x z^{2}+a x^{3} z$ & $3<p$ & 11 \\
\hline $\mathrm{S}_{12}$ & $x^{2} y+y^{2} z+x z^{3}+a z^{5}$ & $3<p$ & 12 \\
\hline $\mathrm{T}_{q, r, s}$ & $\begin{array}{l}x^{q}+y^{r}+z^{s}+a x y z \\
x^{3}+y^{3}+z^{4}+a x y z^{2}\end{array}$ & $\begin{array}{l}3 \leq q \leq r \leq s<p, \frac{1}{q}+\frac{1}{r}+\frac{1}{s}<1 \\
3<p\end{array}$ & $\begin{array}{l}q+r+s-1 \\
12\end{array}$ \\
\hline & & & \\
\hline
\end{tabular}

TABLE 3. Unimodal singularities $(p>2, n=3)$

\begin{tabular}{ll}
\hline Normal form \\
\hline$g\left(x_{1}, x_{2}, x_{3}\right)+x_{4}^{2}+\ldots+x_{n}^{2} \quad g$ is one of the singularities in Table $[3$ \\
\hline
\end{tabular}

TABLE 4. Unimodal singularities $(p>2, n>3)$

\subsection{Right bimodal singularities.}

Theorem 3.3. Let $p=\operatorname{char}(K)>2$. A hypersurface singularity $f \in \mathfrak{m}^{2}$ is right bimodal if and only if it is right equivalent to one of the following forms

I. $\mathbf{n}=\mathbf{1}(f \in K[[x]])$. The list is given in Table 6 .

II. $\mathbf{n}=\mathbf{2}(f \in K[[x, y]])$. The list is given in Table?

III. $\mathbf{n}=\mathbf{3}(f \in K[[x, y, z]])$. The list is given in Table 8 , 


\begin{tabular}{clll}
\hline Name & Normal form & Conditions & $\mu$ \\
\hline $\mathrm{A}_{2}$ & $a x_{1}^{2}+x_{1}^{3}+x_{2} x_{3}+\ldots+x_{n-1} x_{n}$ & $a \in K$ & 2 \\
\hline
\end{tabular}

TABLE 5. Unimodal singularities $(p=2, n \geq 1)$

$I V . \mathbf{n}>\mathbf{3}$. The list is given in Table 9.

Theorem 3.4. Let $p=\operatorname{char}(K)=2$. A hypersurface singularity $f \in \mathfrak{m}^{2}$ is right bimodal if and only if it is right equivalent to one of the following forms

I. $n$ odd: The list is given in the Table 10.

II. $n$ even: The list is given in the Table 11 .

\begin{tabular}{clll}
\hline Name & Normal form & Conditions & $\mu$ \\
\hline $\mathrm{A}_{k}$ & $a_{1} x^{p}+a_{2} x^{2 p}+x^{k+1}$ & $2 p \leq k \leq 3 p-2$ & $k$ \\
\hline
\end{tabular}

TABLE 6. Bimodal singularities $(p>2, n=1)$

\begin{tabular}{clll}
\hline Name & Normal form $\left(\mathbf{a}=a_{0}+a_{1} y\right)$ & Conditions & $\mu$ \\
\hline $\mathrm{A}_{k}$ & $x^{2}+a_{1} y^{p}+a_{2} y^{2 p}+y^{k+1}$ & $2 p \leq k \leq 3 p-2$ & $k$ \\
$\mathrm{D}_{2 p}$ & $x^{2} y+a y^{p}+y^{2 p-1}$ & $3 \leq p$ & $2 p$ \\
$\mathrm{D}_{k}$ & $x^{2} y+a_{1} y^{p}+a_{2} y^{2 p}+y^{k-1}$ & $2 p<k-1 \leq 3 p-1$ & $k$ \\
$\mathrm{E}_{12}$ & $x^{3}+a y^{5}+y^{7}+b x y^{5}$ & $p=5$ & 12 \\
$\mathrm{E}_{13}$ & $x^{3}+x y^{5}+\mathbf{a} y^{7}$ & $p=7$ & 13 \\
$\mathrm{E}_{14}$ & $x^{3}+y^{8}+a y^{7}+b x y^{6}$ & $p=7$ & 14 \\
$\mathrm{E}_{18}$ & $x^{3}+y^{10}+\mathbf{a} x y^{7}$ & $7<p$ & 18 \\
$\mathrm{E}_{19}$ & $x^{3}+x y^{7}+\mathbf{a} y^{11}$ & $7<p$ & 19 \\
$\mathrm{E}_{20}$ & $x^{3}+y^{11}+\mathbf{a} x y^{8}$ & $11<p$ & 20 \\
$\mathrm{~J}_{10}=\mathrm{J}_{2,0}=\mathrm{T}_{2,3,6}$ & $x^{3}+b x^{2} y^{2}+y^{6}+a y^{5}$ & $4 b^{3}+27 \neq 0, p=5$ & 10 \\
$\mathrm{~J}_{2, q}=\mathrm{T}_{2,3,6+q}$ & $x^{3}+x^{2} y^{2}+a y^{p}+b y^{6+q}$ & $p<6+q<2 p, b \neq 0, p \geq 5$ & $q+10$ \\
$\mathrm{~J}_{3,0}$ & $x^{3}+b x^{2} y^{3}+c x y^{7}+y^{9}$ & $4 b^{3}+27 \neq 0,7<p$ & 16 \\
$\mathrm{~J}_{3, q}$ & $x^{3}+x^{2} y^{3}+\mathbf{a} y^{9+q}$ & $a_{0} \neq 0,9<9+q<p$ & $q+16$ \\
$\mathrm{~W}_{17}$ & $x^{4}+x y^{5}+\mathbf{a} y^{7}$ & $7<p$ & 17 \\
$\mathrm{~W}_{18}$ & $x^{4}+y^{7}+\mathbf{a} x^{2} y^{4}$ & $a_{0}^{2} \neq 4,5<p$ & 18 \\
$\mathrm{~W}_{1,0}$ & $x^{4}+\mathbf{a} x^{2} y^{3}+y^{6}$ & $a_{0} \neq 0,7 \leq 6+q<p$ & 15 \\
$\mathrm{~W}_{1, q}$ & $x^{4}+x^{2} y^{3}+\mathbf{a} y^{6+q}$ & $a_{0} \neq 0,5 \leq 4+q<p$ & $2 q+15$ \\
$\mathrm{~W}_{1,2 q-1}^{\sharp}$ & $\left(x^{2}+y^{3}\right)^{2}+\mathbf{a} x y^{4+q}$ & $a_{0} \neq 0,4 \leq 3+q<p>5$ & $2 q+15$ \\
$\mathrm{~W}_{1,2 q}^{\sharp}$ & $\left(x^{2}+y^{3}\right)^{2}+\mathbf{a} x^{2} y^{3+q}$ & $p=5$ & 12 \\
$\mathrm{Z}_{12}$ & $x^{3} y+x y^{4}+a y^{5}+b x^{2} y^{3}$ & $p=5$ & 13 \\
$\mathrm{Z}_{13}$ & $x^{3} y+y^{6}+a y^{5}+b x y^{5}$ & $7<p$ & 17 \\
$\mathrm{Z}_{17}$ & $x^{3} y+\mathbf{a} x y^{6}+y^{8}$ & $7<p$ & 18 \\
$\mathrm{Z}_{18}$ & $x^{3} y+x y^{6}+\mathbf{a} y^{9}$ & $7<p$ & 19 \\
$\mathrm{Z}_{19}$ & $x^{3} y+y^{9}+\mathbf{a} x y^{7}$ & $4 b^{3}+27 \neq 0,7<p$ & 15 \\
$\mathrm{Z}_{1,0}$ & $x^{3} y+b x^{2} y^{3}+c x y^{6}+y^{7}$ & $a_{0} \neq 0,7<7+q<p$ & $q+15$ \\
$\mathrm{Z}_{1, q}$ & $x^{3} y+x^{2} y^{3}+\mathbf{a} y^{7+q}$ & & \\
& & &
\end{tabular}

TABLE 7. Bimodal singularities $(p>2, n=2)$ 


\begin{tabular}{clll}
\hline Name & Normal form $\left(\mathbf{a}=a_{0}+a_{1} y\right)$ & Conditions & $\mu$ \\
\hline & $g(x, y)+z^{2}$ & $g$ one of the series in Table 7 & $\mu(g)$ \\
$\mathrm{Q}_{16}$ & $x^{3}+y z^{2}+y^{7}+\mathbf{a} x y^{5}$ & $7<p$ & 16 \\
$\mathrm{Q}_{17}$ & $x^{3}+y z^{2}+y^{7}+\mathbf{a} y^{8}$ & $7<p$ & 17 \\
$\mathrm{Q}_{18}$ & $x^{3}+y z^{2}+y^{8}+\mathbf{a} x y^{6}$ & $7<p$ & 18 \\
$\mathrm{Q}_{2,0}$ & $x^{3}+y z^{2}+\mathbf{a} x^{2} y^{2}+x y^{4}$ & $a_{0}^{2} \neq 4,3<p$ & 14 \\
$\mathrm{Q}_{2, q}$ & $x^{3}+y z^{2}+x^{2} y^{2}+\mathbf{a} y^{6+q}$ & $a_{0} \neq 0,7 \leq 6+q<p$ & $q+14$ \\
$\mathrm{~S}_{16}$ & $x^{2} z+y z^{2}+x y^{4}+\mathbf{a} y^{6}$ & $5<p$ & 16 \\
$\mathrm{~S}_{17}$ & $x^{2} z+y z^{2}+y^{6}+\mathbf{a} z y^{4}$ & $5<p$ & 17 \\
$\mathrm{~S}_{1,0}$ & $x^{2} y+y z^{2}+y^{5}+\mathbf{a} z y^{3}$ & $a_{0}^{2} \neq 4,3<p$ & 14 \\
$\mathrm{~S}_{1, q}$ & $x^{2} y+y z^{2}+x^{2} y^{2}+\mathbf{a} y^{5+q}$ & $a_{0} \neq 0,5<5+q<p$ & $q+14$ \\
$\mathrm{~S}_{1,2 q-1}$ & $x^{2} y+y z^{2}+z y^{3}+\mathbf{a} x y^{3+q}$ & $a_{0} \neq 0,3<3+q<p$ & $2 q+13$ \\
$\mathrm{~S}_{1,2 q}^{\sharp}$ & $x^{2} y+y z^{2}+z y^{3}+\mathbf{a} x^{2} y^{2+q}$ & $a_{0} \neq 0,3 \leq 2+q<p$ & $2 q+14$ \\
$\mathrm{~T}_{q, r, s}$ & $x^{q}+y{ }^{r}+z^{s}+a x y z+b z^{p}$ & $3 \leq q \leq r<p<s<2 p$ & $q+r+s-1$ \\
$\mathrm{U}_{16}$ & $x^{3}+x z^{2}+y^{5}+\mathbf{a} x^{2} y^{2}$ & $5<p$ & 16 \\
$\mathrm{U}_{1,0}$ & $x^{3}+x z^{2}+x y^{3}+\mathbf{a} y^{3} z$ & $a_{0}\left(a_{0}^{2}+1\right) \neq 0,5<p$ & 14 \\
$\mathrm{U}_{1,2 q-1}$ & $x^{3}+x z^{2}+x y^{3}+\mathbf{a} y^{1+q} z^{2}$ & $a_{0} \neq 0,2 \leq 1+q<p>3$ & $2 q+13$ \\
$\mathrm{U}_{1,2 q}$ & $x^{3}+x z^{2}+x y^{3}+\mathbf{a} y^{3+q} z$ & $a_{0} \neq 0,3<3+q<p$ & $2 q+14$ \\
\hline
\end{tabular}

TABLE 8. Bimodal singularities $(p>2, n=3)$

\begin{tabular}{lll}
\hline Normal form & Condition & $\mu$ \\
\hline$g\left(x_{1}, x_{2}, x_{3}\right)+x_{4}^{2}+\ldots+x_{n}^{2}$ & $g$ is one of the singularities in Table 8 & $\mu(g)$ \\
\hline
\end{tabular}

TABLE 9. Bimodal singularities $(p>2, n>3)$

\begin{tabular}{clll}
\hline Name & Normal form & Conditions & $\mu$ \\
\hline $\mathrm{A}_{4}$ & $a_{1} x_{1}^{2}+a_{2} x_{1}^{4}+x_{1}^{5}+x_{2} x_{3}+\ldots+x_{n-1} x_{n}$ & $a_{1}, a_{2} \in K$ & 4 \\
\hline
\end{tabular}

TABLE 10. Bimodal singularities $(p=2, n$ odd $)$

\begin{tabular}{clc}
\hline Name & Normal form & $\mu$ \\
\hline $\mathrm{D}_{4}$ & $a_{1} x_{1}^{2}+a_{2} x_{2}^{2}+x_{1}^{3}+x_{2}^{3}+x_{3} x_{4}+\ldots+x_{n-1} x_{n}$ & 4 \\
$\mathrm{D}_{6}$ & $a_{1} x_{1}^{2}+a_{2} x_{2}^{2}+x_{1}^{2} x_{2}+x_{1} x_{2}^{3}+x_{3} x_{4}+\ldots+x_{n-1} x_{n}$ & 6 \\
$\mathrm{E}_{7}$ & $a_{1} x_{1}^{2}+a_{2} x_{2}^{2}+x_{1}^{3}+x_{1} x_{2}^{3}+x_{3} x_{4}+\ldots+x_{n-1} x_{n}$ & 7 \\
$\mathrm{E}_{8}$ & $a_{1} x_{1}^{2}+a_{2} x_{2}^{2}+x_{1}^{3}+x_{2}^{5}+x_{3} x_{4}+\ldots+x_{n-1} x_{n}$ & 8 \\
\hline
\end{tabular}

TABLE 11. Bimodal singularities $(p=2, n$ even)

3.3. Adjacencies of simple, unimodal and bimodal singularities. In the following we give diagrams of adjacencies for all class of simple singularities and singularities in Tables 1-11. Moreover a singularity in these tables deforms only into classes listed in the diagrams. Recall that a class $\mathcal{D}$ of singularities is adjacent to class $\mathcal{C}, \mathcal{C} \leftarrow \mathcal{D}$, if every $f \in \mathcal{D}$ can be deformed into an element in $\mathcal{C}$ by a deformation. That is, there exists an unfolding $f_{t}$ of $f=f_{t_{0}}$ over an affine variety $T, t_{0}$ and a Zariski open subset $V \subset T$ such that $f_{t} \in \mathcal{C}$ for all $t \in V$.

Theorem 3.5. Any singularity in Tables 1-11 deforms only into singularities given in the following adjacency diagrams $\left(\mathrm{T}_{2,3,6+q}=\mathrm{J}_{2, q}, \mathrm{~T}_{2,4,4+q}=\mathrm{X}_{1, q}, \mathrm{~T}_{2,4+r, 4+s}=\mathrm{Y}_{r, s}\right)$ : 
- $A_{k-1}<D_{k}<E_{k+1}$
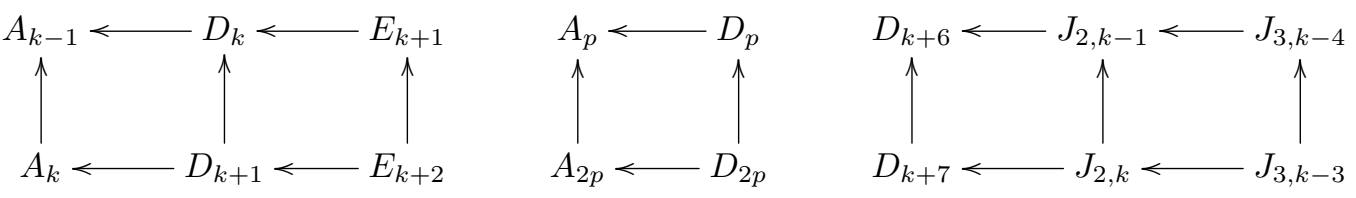

- $E_{8} \leftarrow J_{2,0} ; E_{14} \leftarrow J_{3,0} ; J_{s, k} \leftarrow E_{6 s+k-1} ; s=2,3 ; k=1,2,3$.

- $T_{q^{\prime}, r^{\prime}, s^{\prime}} \leftarrow T_{q, r, s}$ if $\left(q^{\prime}, r^{\prime}, s^{\prime}\right) \leq(q, r, s)$, i.e. $q^{\prime} \leq q, r^{\prime} \leq r, s^{\prime} \leq s$.

- $Q_{12} \longleftarrow Q_{2,0} \longleftarrow Q_{2,1} \longleftarrow Q_{2,2} \longleftarrow Q_{2,3} \longleftarrow \cdots$

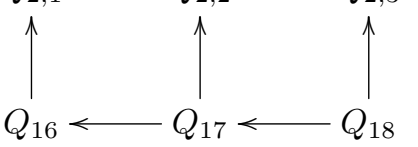

-
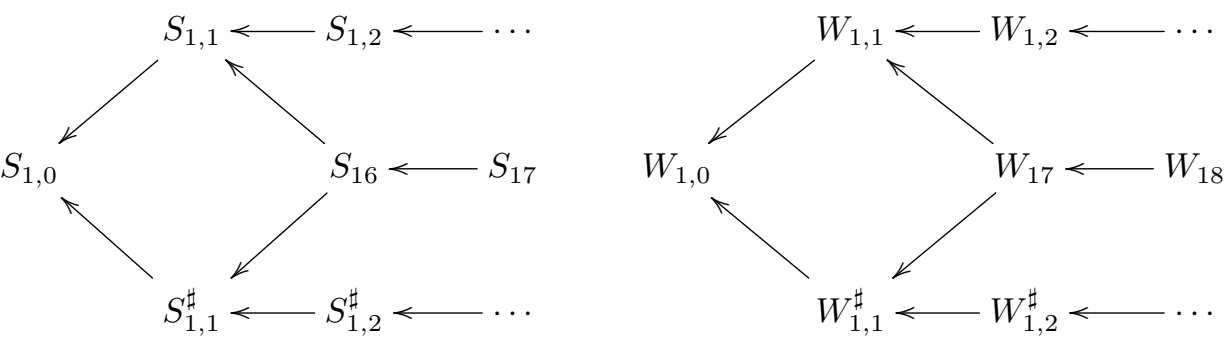

- $E_{6} \longleftarrow T_{3,3,3} \longleftarrow T_{3,3,4} \longleftarrow Q_{10} \longleftarrow Q_{11} \longleftarrow Q_{12}$
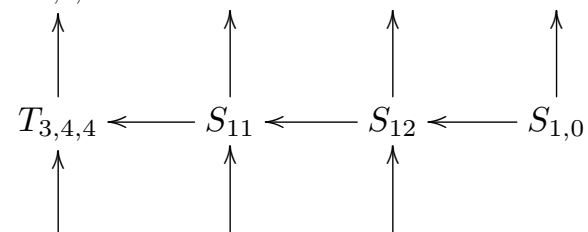

$T_{4,4,4}<U_{12} \leftarrow U_{1,0}$

- $Z_{13} \longleftarrow Z_{1,0} \longleftarrow Z_{1,1} \longleftarrow Z_{1,2} \longleftarrow Z_{1,3} \longleftarrow \cdots$

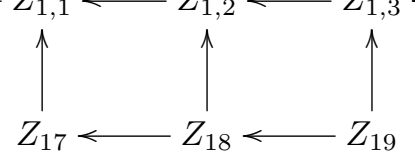

- $E_{7} \longleftarrow T_{2,4,4} \longleftarrow T_{2,4,5} \longleftarrow Z_{11} \longleftarrow Z_{12} \longleftarrow Z_{13}$

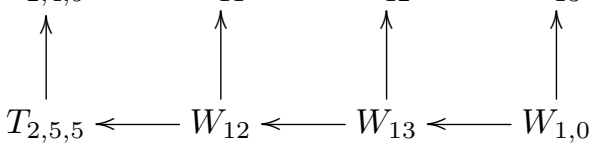

3.4. Milnor number, $\mu$-contant stratum and proper modality. In this section we give several applications of the classification of unimodal and bimodal singularities. The first two corollaries below follow from the classification of right simple, unimodal and bimodal singularities ([14, and Theorems 3.1 3.4 . The remainings will be proved in Section 5.5

Let $f \in K[[\mathbf{x}]]$, with $p=\operatorname{char}(K)>0$ such that $\operatorname{rmod}(f) \leq 2$. Then

Corollary 3.6. If $p \leq 3$, then $f$ is of type $A, D$ or $E$.

In [14, using the classification of right simple singularities, we showed that, if $f$ is right simple, then $\mu(f) \leq p$. We also conjectured ([14, Conjecture 3.5) that, for any sequence $f_{k} \in K\left[\left[x_{1}, \ldots, x_{n}\right]\right]$ of isolated singularities, if $\mu\left(f_{k}\right)$ goes to infinity as $k \rightarrow \infty$, then so does $\operatorname{rmod}\left(f_{k}\right)$. In this section we give an affirmative answer for the conjecture, namely

Corollary 3.7. We have $\mu(f) \leq 4 p$.

Corollary 3.8. The $\mu$-constant stratum of $f$ is a linear space, and hence smooth.

Note that this is not true in general as shown by Luengo in [17. 
Corollary 3.9. We have $\operatorname{rmod}(f)=\operatorname{pmod}(f)$.

\section{Singularity Determinator}

In 6] Arnol'd supplied lists of normal forms which contain all the singularities with the modality number $\bmod =0,1,2$, all the singularities with Milnor number $\mu \leq 16$, all the singularities of corank 2 with nonzero 4-jet, all the singularities of corank 3 with a 3 -jet, which determine an irreducible cubic, and some other singularities. The proof of Arnol'd is organized as a determinator consisting of 105 theorems. We follow this scheme and organize our proof a singularity determinator of 152 theorems. This gives an algorithm finding for every given sigularity its place in the list of $\S 3$. However we obtain only the classification of simple, unimodal and bimodal singularities. For the other classifications a new method should be needed.

Notations:

$\begin{array}{ll}\stackrel{ }{\mapsto} & \text { "implies". } \\ \mapsto & \text { "see". }\end{array}$

crk the corank of the Hessian of $f$ at the origin, which is used to reduce the number of variables, see 5 .

$\Delta \quad$ discriminant, in Theorems 71, 72; $\Delta=4\left(a^{3}+b^{3}\right)+27-a^{2} b^{2}-18 a b$.

$j_{\left\{\mathbf{x}^{\left.\alpha_{i}\right\}}\right.} f(\mathbf{x})$ quasijet of $f$ determined by $\left\{\mathbf{x}^{\alpha_{i}}\right\}$, defined as follows.

Here $\left\{\alpha_{i}\right\}$ is a system of $n$ points defining an affine hyperplane $H$ in $\mathbb{R}^{n}$. Let $v: \mathbb{R}^{n} \rightarrow \mathbb{R}$ be the linear form defining $H$ with $v\left(\alpha_{i}\right)=1$ for all $i$. Then $j_{\left\{\mathbf{x}^{\alpha_{i}}\right\}} f$ is the image of $f$ in $K[[\mathbf{x}]]$ modulo the ideal generated by $x^{\alpha}, v(\alpha)>1$.

\subsection{Singularity determinator in characteristic $\geq 5$.}

1. $\mu(f)<\infty \Rightarrow$ one of the four possibilities holds:

$$
\begin{aligned}
\operatorname{crk}(f) & \leq 1 \\
= & \mapsto \mathbf{2}, \\
& =3 \mapsto \mathbf{4}-\mathbf{7 3}, \\
> & \mapsto \mapsto \mathbf{7 4 - 1 9},
\end{aligned}
$$

2. $\operatorname{crk}(f) \leq 1, \mu<3 p \Rightarrow \operatorname{rmod}(f)=\lfloor\mu / p\rfloor$ and $f \in A_{\mu}$.

3. $\operatorname{crk}(f) \leq 1, \mu \geq 3 p \Rightarrow \operatorname{rmod}(f) \geq 3$.

\section{Corank 2 Singularities}

Through theorems $\mathbf{4}-\mathbf{7 3}, f \in K[[x, y]]$.

4. $j^{2}(f)=0 \Rightarrow$ one of the four possibilities holds:

$$
\begin{array}{rll}
j^{3} f & \sim_{r} x^{2} y+y^{3} & \mapsto \mathbf{5}, \\
& \sim_{r} x^{2} y & \mapsto \mathbf{6}, \\
& \sim_{r} x^{3} & \mapsto \mathbf{9}-\mathbf{3 0}, \\
= & 0 & \mapsto \mathbf{3 1 - 7 3 .}
\end{array}
$$

5. $j^{3}(f)=x^{2} y+y^{3} \Rightarrow f \in D_{4}$.

6. $j^{3}(f)=x^{2} y \Rightarrow f \sim_{r} x^{2} y+\alpha(y), j^{3}(\alpha)=0 \mapsto \mathbf{7 - 8}$.

7. $f=x^{2} y+\alpha(y), j^{3}(\alpha)=0, k:=\mu(\alpha) \leq 3 p-1 \Rightarrow f \in D_{k+2}$.

8. $f=x^{2} y+\alpha(y), j^{3}(\alpha)=0, \mu(\alpha) \geq 3 p \Rightarrow \operatorname{rmod}(f) \geq 3$.

Through theorems 9-12, $k=1,2,3$ for $p>7, k=1,2$ for $p=7, k=1$ for $p=5$.

9. $j_{x^{3}, y^{3 k}} f(x, y)=x^{3} \Rightarrow$ one of the four possibilities holds:

$$
\begin{array}{lll}
j_{x^{3}, y^{3 k+1}} f(x, y) & \sim_{r} x^{3}+y^{3 k+1} & \mapsto \mathbf{1 0}, \\
j_{x^{3}, x y^{2 k+1}} f(x, y) & \sim_{r} x^{3}+x y^{2 k+1} & \mapsto \mathbf{1 1}, \\
j_{x^{3}, y^{3 k+2}} f(x, y) & \sim_{r} x^{3}+y^{3 k+2} & \mapsto \mathbf{1 2}, \mathbf{1 3}, \\
j_{x^{3}, y^{3 k+2}} f(x, y) & =x^{3} & \mapsto \mathbf{1 3}, \mathbf{2 6} .
\end{array}
$$

10. $j_{x^{3}, y^{3 k+1}} f(x, y)=x^{3}+y^{3 k+1}$ and $3 k+1<p \Rightarrow f \in E_{6 k}$.

11. $j_{x^{3}, x y^{2 k+1}} f(x, y)=x^{3}+x y^{2 k+1}$ and $3 k+1<p \Rightarrow f \in E_{6 k+1}$.

12. $j_{x^{3}, y^{3 k+2}} f(x, y)=x^{3}+y^{3 k+2}$ and $3 k+2<p \Rightarrow f \in E_{6 k+2}$. 
13. $p=5$ and $j_{x^{3}, y^{5}} f(x, y)=x^{3}+a y^{5} \Rightarrow$ one of the three possibilities holds:

$$
\begin{aligned}
& j_{x^{3}, y^{6}} f(x, y) \quad \sim_{r} \quad x^{3}+b x^{2} y^{2}+y^{6}+a y^{5}, 4 b^{3}+27 \neq 0 \quad \mapsto \mathbf{1 4}, \\
& \sim_{r} x^{3}+x^{2} y^{2}+a y^{5} \quad \mapsto \mathbf{1 5}, \mathbf{1 6}, \\
& \sim_{r} x^{3}+a y^{5} \quad \mapsto 17 .
\end{aligned}
$$

14. $p=5$ and $j_{x^{3}, y^{6}} f=x^{3}+b x^{2} y^{2}+y^{6}+a y^{5}, 4 b^{3}+27 \neq 0 \Rightarrow f \in J_{2,0}$.

15. $p=5, j_{x^{3}, y^{6}} f=x^{3}+x^{2} y^{2}+a y^{5}$ and $\mu<14 \Rightarrow f \in J_{2, q}$ with $q=\mu-10>0$.

16. $p=5, j_{x^{3}, y^{6}} f=x^{3}+x^{2} y^{2}+a y^{5}$ and $\mu \geq 14 \Rightarrow \operatorname{rmod}(f) \geq 3$.

17. $p=5, j_{x^{3}, y^{6}} f=x^{3}+a y^{5} \Rightarrow$ one of the two possibilities holds:

$$
\begin{array}{llll}
j_{x^{3}, y^{7}} f(x, y) & \sim_{r} x^{3}+a y^{5}+y^{7} & \mapsto \mathbf{1 8} \\
j_{x^{3}, y^{7}} f(x, y) & =x^{3}+a y^{5} & \mapsto \mathbf{1 9} .
\end{array}
$$

18. $p=5, j_{x^{3}, y^{7}} f=x^{3}+a y^{5}+y^{7} \Rightarrow f \in E_{12}$.

19. $p=5, j_{x^{3}, y^{7}} f=x^{3}+a y^{5} \Rightarrow \operatorname{rmod}(f) \geq 3$.

20. $p=7$ and $j_{x^{3}, y^{7}} f=x^{3}+a y^{7} \Rightarrow$ one of the three possibilities holds:

$$
\begin{array}{llll}
j_{x^{3}, x y^{5}} f(x, y) & \sim_{r} x^{3}+x y^{5}+a y^{7} & \mapsto \mathbf{2 1}, \\
j_{x^{3}, y^{8}} f(x, y) & \sim_{r} x^{3}+y^{8}+a y^{7} & \mapsto \mathbf{2 2}, \\
j_{x^{3}, y^{8}} f(x, y) & =x^{3}+a y^{7} & \mapsto \mathbf{2 3} .
\end{array}
$$

21. $p=7$ and $j_{x^{3}, x y^{5}} f=x^{3}+x y^{5}+a y^{7} \Rightarrow f \in E_{13}$.

22. $p=7$ and $j_{x^{3}, y^{8}} f=x^{3}+y^{8}+a y^{7} \Rightarrow f \in E_{14}$.

23. $p=7$ and $j_{x^{3}, y^{8}} f=x^{3}+a y^{7} \Rightarrow \operatorname{rmod}(f) \geq 3$.

24. $p=11$ and $j_{x^{3}, y^{11}} f=x^{3}+y^{11} \Rightarrow \operatorname{rmod}(f) \geq 3$.

25. $j_{x^{3}, y^{11}} f(x, y)=x^{3} \Rightarrow f \in\left\langle x, y^{4}\right\rangle^{3} \Rightarrow \operatorname{rmod}(f) \geq 3$.

Through theorems 26-29, $k=2,3$.

26. $j_{x^{3}, y^{3 k-1}} f(x, y)=x^{3} \Rightarrow$ one of the three possibilities holds:

$$
\begin{aligned}
& j_{x^{3}, y^{3 k}} f(x, y) \quad \sim_{r} \quad x^{3}+a x^{2} y^{k}+y^{3 k}, 4 a^{3}+27 \neq 0 \quad \mapsto \mathbf{2 7}, \\
& \sim_{r} x^{3}+x^{2} y^{k} \quad \mapsto \mathbf{2 8}, \mathbf{2 9}, \\
& \begin{array}{lll}
\sim_{r} x^{3} & \mapsto \mathbf{9}, \mathbf{3 0} .
\end{array}
\end{aligned}
$$

27. $j_{x^{3}, y^{3 k}} f(x, y)=x^{3}+a x^{2} y^{k}+y^{3 k}, 4 a^{3}+27 \neq 0$ and $3 k<p \Rightarrow f \in J_{k, 0}$

28. $j_{x^{3}, y^{3 k}} f(x, y)=x^{3}+x^{2} y^{k}, 3 k<p$ and $\mu-3 k+2<2 p \Rightarrow f \in J_{k, q}$ with $q=\mu-6 k+2$.

29. $j_{x^{3}, y^{3 k}} f(x, y)=x^{3}+x^{2} y^{k}, 3 k<p$ and $\mu-3 k+2 \geq 2 p \Rightarrow \operatorname{rmod}(f) \geq 3$.

30. $p=7$ and $j_{x^{3}, y^{6}} f(x, y)=x^{3}+x^{2} y^{2}$ and $\mu<18 \Rightarrow f \in J_{2, q}$ with $q=\mu-10>0$.

\section{Series X}

31. $j^{3} f=0 \Rightarrow$ one of the six possibilities holds:

$$
\begin{aligned}
& j^{4} f \quad \sim_{r} \quad x^{4}+a x^{2} y^{2}+y^{4}, a^{2}+4 \neq 0 \quad \mapsto \quad \mathbf{3 2}, \\
& \sim_{r} x^{4}+x^{2} y^{2} \quad \mapsto \mathbf{3 3}, \mathbf{3 4}, \\
& \sim_{r} \quad x^{2} y^{2} \quad \mapsto \quad 35-38, \\
& \sim_{r} x^{3} y \quad \mapsto \mathbf{3 9}, \\
& \sim_{r} x^{4} \quad \mapsto 54, \\
& =0 \quad \mapsto 71 .
\end{aligned}
$$

32. $j^{4}(f)=x^{4}+a x^{2} y^{2}+y^{4}, a^{2}+4 \neq 0 \Rightarrow f \in X_{9}$.

33. $j^{4}(f)=x^{4}+x^{2} y^{2}$ and $\mu(f)<2 p+5 \Rightarrow f \in X_{1, q}$.

34. $j^{4}(f)=x^{4}+x^{2} y^{2}$ and $\mu(f) \geq 2 p+5 \Rightarrow \operatorname{rmod}(f) \geq 3$.

35. $j^{4}(f)=x^{2} y^{2} \Rightarrow f=f_{1} \cdot f_{2}$ with $\operatorname{mt}\left(f_{1}\right)=\operatorname{mt}\left(f_{2}\right)=2$ and $2 \leq \mu\left(f_{1}\right) \leq \mu\left(f_{2}\right) \Rightarrow \mathbf{3 6}$.

Through theorems 36-39, $1 \leq r:=\mu\left(f_{1}\right)-1 \leq s:=\mu\left(f_{2}\right)-1$.

36. $\mu\left(f_{1}\right) \geq p$ or $\mu\left(f_{2}\right) \geq 2 p \Rightarrow \operatorname{rmod}(f) \geq 3$.

37. $\mu\left(f_{2}\right)<p \Rightarrow \operatorname{rmod}(f)=1$ and $f \in Y_{r, s}$.

38. $\mu\left(f_{1}\right)<p$ and $p \leq \mu\left(f_{2}\right)<2 p \Rightarrow \operatorname{rmod}(f)=2$ and $f \in Y_{r, s}$.

39. $j^{4}(f)=x^{3} y \Rightarrow j_{x^{3} y, y^{4}} f=x^{3} y \mapsto 40,44$.

\section{Series Z}

Through theorems 40-43, $5<p$ and $q=1,2$.

40. $j_{x^{3} y, y^{3 q+1}} f=x^{3} y \Rightarrow$ one of the four possibilities holds: 


$$
\begin{array}{llll}
j_{x^{3} y, y^{3 q+2}} f & \sim_{r} & x^{3} y+y^{3 q+2} & \mapsto \mathbf{4 1}, \\
j_{x^{3} y, x y^{2 q+2}} f & \sim_{r} \quad x^{3} y+x y^{2 q+2} & \mapsto \mathbf{4 2}, \\
j_{x^{3} y, y^{3 q+3}} f & \sim_{r} & x^{3} y+y^{3 q+3} & \mapsto \mathbf{4 3}, \\
j_{x^{3} y, y^{3 q+3}} f & = & x^{3} y & \mapsto \mathbf{4 9 - 5 3} .
\end{array}
$$

41. $j_{x^{3} y, y^{3 q+2}} f=x^{3} y+y^{3 q+2}, 3 q+2<p \Rightarrow f \in Z_{6 q+5}$.

42. $j_{x^{3} y, x y^{2 q+2}} f=x^{3} y+x y^{2 q+2}, 3 q+3<p \Rightarrow f \in Z_{6 q+6}$.

43. $j_{x^{3} y, y^{3 q+3}} f=x^{3} y+y^{3 q+3}, 3 q+3<p \Rightarrow f \in Z_{6 q+7}$.

44. $p=5$ and $j_{x^{3} y, y^{4}} f=x^{3} y \Rightarrow$ one of the three possibilities holds:

$$
\begin{array}{lll}
j_{x^{3} y, x y^{4}} f & \sim_{r} x^{3} y+x y^{4}+a y^{5} & \mapsto \mathbf{4 5}, \\
j_{x^{3} y, y^{6}} f & \sim_{r} x^{3} y+y^{6}+a y^{5} & \mapsto \mathbf{4 6}, \\
j_{x^{3} y, y^{6}} f=x^{3} y+a y^{5} & \mapsto \mathbf{4 7 .}
\end{array}
$$

45. $p=5$ and $j_{x^{3} y, x y^{4}} f=x^{3} y+x y^{4}+a y^{5} \Rightarrow f \in Z_{12}$.

46. $p=5$ and $j_{x^{3} y, x y^{4}} f=x^{3} y+y^{6}+a y^{5} \Rightarrow f \in Z_{13}$.

47. $p=5$ and $j_{x^{3} y, x y^{4}} f=x^{3} y+a y^{5} \Rightarrow \operatorname{rmod}(f) \geq 3$.

48. $p=7$ and $j_{x^{3} y, y^{7}} f=x^{3} y \Rightarrow \operatorname{rmod}(f) \geq 3$.

49. $j_{x^{3} y, y^{6}} f=x^{3} y \Rightarrow$ one of the three possibilities holds:

$$
\begin{aligned}
& j_{x^{3} y, y^{7}} f=y\left(x^{3}+b x^{2} y^{2}+y^{9}\right), 4 b^{3}+27 \neq 0 \mapsto \mathbf{5 0} \\
& =y\left(x^{3}+x^{2} y^{2}\right) \quad \mapsto \mathbf{5 1 , 5 2} \\
& =x^{3} y \quad \mapsto 53 .
\end{aligned}
$$

50. $j_{x^{3} y, y^{7}} f=y\left(x^{3}+b x^{2} y^{2}+y^{9}\right), 4 b^{3}+27 \neq 0 \Rightarrow f \in Z_{1,0}$.

51. $j_{x^{3} y, y^{7}} f=y\left(x^{3}+x^{2} y^{2}\right)$ and $\mu-8<p \Rightarrow f \in Z_{1, r}$ with $r=\mu-15>0$.

52. $j_{x^{3} y, y^{7}} f=y\left(x^{3}+x^{2} y^{2}\right)$ and $\mu-8 \geq p \Rightarrow \operatorname{rmod}(f) \geq 3$.

53. $j_{x^{3} y, y^{9}} f=x^{3} y \Rightarrow f \in\langle y\rangle \cdot\left\langle x, y^{3}\right\rangle^{3} \Rightarrow \operatorname{rmod}(f) \geq 3$.

\section{Series W}

Through theorems $\mathbf{5 4 - 5 6}, 5<p$.

54. $j^{4} f=x^{4} \Rightarrow j_{x^{4}, y^{4}} f=x^{4} \Rightarrow$ one of the three possibilities holds:

$$
\begin{array}{llll}
j_{x^{4}, y^{5}} f & \sim_{r} & x^{4}+y^{5} & \mapsto \mathbf{5 5}, \\
j_{x^{4}, x y^{4}} f & \sim_{r} & x^{4}+x y^{4} & \mapsto \mathbf{5 6}, \\
j_{x^{4}, x y^{4}} f= & x^{4} & \mapsto \mathbf{5 7 , 6 0 .} .
\end{array}
$$

55. $j_{x^{4}, y^{5}} f=x^{4}+y^{5} \Rightarrow f \in W_{12}$.

56. $j_{x^{4}, x y^{4}} f=x^{4}+x y^{4} \Rightarrow f \in W_{13}$.

57. $p=5$ and $j_{x^{4}, y^{4}} f=x^{4} \Rightarrow$ one of the two possibilities holds:

$$
\begin{array}{lll}
j_{x^{4}, x y^{4}} f & \sim_{r} x^{4}+x y^{4}+a y^{5} & \mapsto \mathbf{5 8} \\
j_{x^{4}, x y^{4}} f=x^{4}+a y^{5} & \mapsto \mathbf{5 9} .
\end{array}
$$

58. $p=5$ and $j_{x^{4}, x y^{4}} f=x^{4}+x y^{4}+a y^{5} \Rightarrow f \in W_{13}$.

59. $p=5$ and $j_{x^{4}, x y^{4}} f=x^{4}+a y^{5} \Rightarrow \operatorname{rmod}(f) \geq 3$.

Through theorems 60-70, $5<p$.

60. $j_{x^{4}, x y^{4}} f=x^{4} \Rightarrow$ one of the four possibilities holds:

$$
\begin{array}{rll}
j_{x^{4}, y^{6} f} & \sim_{r} x^{4}+b x^{2} y^{3}+y^{6}, b^{2} \neq 4 & \mapsto \mathbf{6 1}, \\
& \sim_{r} x^{4}+x^{2} y^{3} & \mapsto \mathbf{6 2 , 6 3}, \\
& \sim_{r}\left(x^{2}+y^{3}\right)^{2} & \mapsto \mathbf{6 4}, \mathbf{6 5} \\
& =x^{4} & \mapsto \mathbf{6 6} .
\end{array}
$$

61. $j_{x^{4}, y^{6}} f=x^{4}+b x^{2} y^{3}+y^{6}, b^{2} \neq 4 \Rightarrow f \in W_{1,0}$.

62. $j_{x^{4}, y^{6}} f=x^{4}+x^{2} y^{3}$ and $\mu-8<p \Rightarrow f \in W_{1, q}(q=\mu-15>0)$.

63. $j_{x^{4}, y^{6}} f=x^{4}+x^{2} y^{3}$ and $\mu-8 \geq p \Rightarrow \operatorname{rmod}(f) \geq 3$.

64. $j_{x^{4}, y^{6}} f=\left(x^{2}+y^{3}\right)^{2}$ and $\mu-8<p \Rightarrow f \in W_{k, q}^{\sharp}(q=\mu-15>0)$.

65. $j_{x^{4}, y^{6}} f=\left(x^{2}+y^{3}\right)^{2}$ and $\mu-8 \geq p \Rightarrow \operatorname{rmod}(f) \geq 3$.

66. $j_{x^{4}, y^{6}} f=x^{4} \Rightarrow$ one of the three possibilities holds: 


$$
\begin{array}{rlll}
j_{x^{4}, x y^{5}} f & \sim_{r} & x^{4}+x y^{5} & \mapsto \mathbf{6 7}, \\
j_{x^{4}, y^{7}} f & \sim_{r} x^{4}+y^{7} & \mapsto \mathbf{6 8}, \mathbf{6 9}, \\
& =x^{4} & \mapsto \mathbf{7 0 .}
\end{array}
$$

67. $j_{x^{4}, x y^{5}} f=x^{4}+x y^{5} \Rightarrow f \in W_{17}$.

68. $j_{x^{4}, y^{7}} f=x^{4}+y^{7}$ and $p>7 \Rightarrow f \in W_{18}$.

69. $p=7$ and $j_{x^{4}, y^{7}} f=x^{4}+y^{7} \Rightarrow \operatorname{rmod}(f) \geq 3$.

70. $j_{x^{4}, y^{7}} f=x^{4} \Rightarrow \operatorname{rmod}(f) \geq 3$.

Through theorems $\mathbf{5 3}-\mathbf{5 5}, 5<p$.

71. $j^{4} f=0 \Rightarrow$ one of the two possibilities holds:

$$
\begin{array}{lclll}
j_{5} f & \sim_{r} \quad x^{4} y+a x^{3} y^{2}+b x^{2} y^{3}+x y^{4}, \Delta \neq 0, a b \neq 9 & \mapsto \mathbf{5 4}, \\
j_{5} f \quad \text { is } \quad \text { degenerate } & \mapsto \mathbf{5 5 .}
\end{array}
$$

72. $j_{5} f=x^{4} y+a x^{3} y^{2}+b x^{2} y^{3}+x y^{4}, \Delta \neq 0, a b \neq 9 \Rightarrow f \sim_{r} x^{4} y+a x^{3} y^{2}+b x^{2} y^{3}+x y^{4}+c x^{3} y^{3}$ with $\Delta \neq 0, a b \neq 9$ and therefore $\operatorname{rmod}(f) \geq 3$.

73. If $j_{5} f$ is degenerate $\Rightarrow \operatorname{rmod}(f) \geq 3$.

\section{Corank 3 Singularities}

Through theorems $\mathbf{7 4 - 1 2 0 ,} f \in K[[x, y, z]]$.

74. $j^{2} f(x, y, z)=0 \Rightarrow$ one of the ten possibilities holds:

$$
\begin{aligned}
& j^{3} f \quad \sim_{r} \quad x^{3}+y^{3}+z^{3}+a x y z, a^{3}+27 \neq 0 \quad \mapsto \quad \mathbf{7 5}, \\
& \sim_{r} x^{3}+y^{3}+x y z \quad \mapsto \mathbf{7 6}, \\
& \sim_{r} x^{3}+x y z \quad \mapsto \mathbf{7 9}, \\
& \sim_{r} x y z \quad \mapsto 80, \\
& \sim_{r} x^{3}+y z^{2} \quad \mapsto \mathbf{8 1}-\mathbf{9 1}, \\
& \sim_{r} x^{2} z+y z^{2} \quad \mapsto 92-106, \\
& \sim_{r} x^{3}+x z^{2} \quad \mapsto 107-117, \\
& \sim_{r} x^{2} y \quad \mapsto \mathbf{1 1 8}, \\
& \sim_{r} x^{3} \quad \mapsto 119, \\
& =0 \quad \mapsto 120 .
\end{aligned}
$$

\section{Series T}

75. $j^{3} f(x, y, z)=x^{3}+y^{3}+z^{3}+a x y z, a^{3}+27 \neq 0 \Rightarrow f \in P_{8}$.

76. $j^{3} f(x, y, z)=x^{3}+y^{3}+x y z \Rightarrow f \sim_{r} x^{3}+y^{3}+x y z+\alpha(z), j^{3} \alpha=0 \mapsto \mathbf{7 7}, \mathbf{7 8}$.

77. $f=x^{3}+y^{3}+x y z+\alpha(z), j^{3} \alpha=0, q:=\mu(\alpha)+1<2 p \Rightarrow f \in P_{q+5}=T_{3,3, q}(q>3)$.

78. $f=x^{3}+y^{3}+x y z+\alpha(z), j^{3} \alpha=0, \mu(\alpha)+1 \geq 2 p \Rightarrow \operatorname{rmod}(f) \geq 3$.

79. $j^{3} f(x, y, z)=x^{3}+x y z \Rightarrow f=x^{3}+x y z+\alpha(y)+\beta(z), j^{3}(\alpha, \beta)=0$ and $q:=\mu(\alpha)+1 \leq r:=$ $\mu(\beta)+1 \Rightarrow$ one of the three possibilities holds:

(i) $r<p \quad \Rightarrow \operatorname{rmod}(f)=1$ and $f \in T_{3, q, r}$,

(ii) $q<p \leq r<2 p \Rightarrow \operatorname{rmod}(f)=2$ and $f \in T_{3, q, r}$,

(iii) otherwise $\Rightarrow \operatorname{rmod}(f) \geq 3$.

80. $j^{3} f(x, y, z)=x y z \Rightarrow f \sim_{r} x y z+\alpha(x)+\beta(y)+\gamma(z), j^{3}(\alpha, \beta, \gamma)=0$ and

$q:=\mu(\alpha)+1 \leq r:=\mu(\beta)+1 \leq s:=\mu(\gamma)+1 \Rightarrow$ one of the three possibilities holds:

(i) $s<p \quad \Rightarrow \operatorname{rmod}(f)=1$ and $f \in T_{q, r, s}$,

(ii) $r<p \leq s<2 p \Rightarrow \operatorname{rmod}(f)=2$ and $f \in T_{q, r, s}$.

(iii) otherwise $\quad \Rightarrow \operatorname{rmod}(f) \geq 3$.

\section{Series Q}

Through theorems 81-91, $\varphi=x^{3}+y z^{2}, j_{\lambda}^{*}=j_{y z^{2}, x^{3}, \lambda},(\lambda$ is a polynomial $)$.

81. $j^{3} f=\varphi \Rightarrow f \sim_{r} \varphi+\alpha(y)+x \beta(y), j^{3}(\alpha, x \beta)=0 \mapsto \mathbf{8 2}$.

Through theorems 82-85, $k=1,2$.

82. $f=\varphi+\alpha(y)+x \beta(y), j_{y^{3 k}}^{*} f=\varphi \Rightarrow$ one of the four possibilities holds: 


$$
\begin{array}{llll}
j_{3^{3 k+1}}^{*} f & \sim_{r} & \varphi+y^{3 k+1} & \mapsto \mathbf{8 3} \\
j_{x y^{2 k+1}}^{*} f & \sim_{r} & \varphi+x y^{2 k+1} & \mapsto \mathbf{8 4} \\
j_{y^{3 k+2}}^{*} f & \sim_{r} & \varphi+y^{3 k+2} & \mapsto \mathbf{8 5}, \mathbf{8 6}, \\
j_{y^{3 k+2}}^{*} f & \sim_{r} \varphi & \mapsto \mathbf{8 7}
\end{array}
$$

83. $j_{y^{3 k+1}}^{*} f=\varphi+y^{3 k+1}$ and $3 k+1<p \Rightarrow f \in Q_{6 k+4}$.

84. $j_{x y^{2 k+1}}^{*} f=\varphi+x y^{2 k+1}$ and $3 k+1<p \Rightarrow f \in Q_{6 k+5}$.

85. $j_{y^{3 k+2}}^{*} f=\varphi+y^{3 k+2}$ and $3 k+2<p \Rightarrow f \in Q_{6 k+6}$.

86. $p=5$ and $j_{x y^{3}}^{*} f=\varphi \Rightarrow \operatorname{rmod}(f) \geq 3$.

87. $f=\varphi+\alpha(y)+x \beta(y), j_{y^{5}}^{*} f=\varphi \Rightarrow$ one of the three possibilities holds:

$$
\begin{array}{rlrl}
j_{y^{6}}^{*} f & \sim_{r} \varphi+a x^{2} y^{2}+x y^{4}, a^{2} \neq 4 & \mapsto \mathbf{8 8}, \\
& \sim_{r} \varphi+x^{2} y^{2} & \mapsto \mathbf{8 9}, \mathbf{9 0}, \\
& =\varphi & & \mapsto \mathbf{9 1}
\end{array}
$$

88. $j_{y^{6}}^{*} f=\varphi+a x^{2} y^{2}+x y^{4}, a^{2} \neq 4 \Rightarrow f \in Q_{2,0}$.

89. $j_{y^{6}}^{*} f=\varphi+x^{2} y^{2}$ and $\mu-5<p \Rightarrow f \in Q_{2, q}(q=\mu-12>0)$.

90. $j_{y^{6}}^{*} f=\varphi+x^{2} y^{2}$ and $\mu-5 \geq p \Rightarrow \operatorname{rmod}(f) \geq 3$.

91. $p=7$ and $j_{y^{6}}^{*} f=\varphi \Rightarrow \operatorname{rmod}(f) \geq 3$.

\section{Series S}

Through theorems 92-106, $\varphi=x^{2} z+y z^{2}, j_{\lambda}^{*}=j_{x^{2} y, y z^{2}, \lambda},(\lambda$ is a polynomial $)$.

92. $j^{3} f=\varphi \Rightarrow f=\varphi+\alpha(y)+x \beta(y)+z \gamma(y), j^{3}(\alpha, x \beta, z \gamma)=0 \mapsto \mathbf{9 3}$.

93. $f=\varphi+\alpha(y)+x \beta(y)+z \gamma(y), j_{y^{3}}^{*} f=\varphi \Rightarrow$ one of the three possibilities holds:

$$
\begin{array}{llll}
j_{y^{4}}^{*} f & \sim_{r} \varphi+y^{4} & \mapsto \mathbf{9 4}, \\
j_{x y^{3}}^{*} f \sim_{r} \varphi+x y^{3} & \mapsto \mathbf{9 5}, \\
j_{x y^{3}}^{*} f=\varphi & \mapsto \mathbf{9 6 , 1 0 6 .}
\end{array}
$$

94. $j_{y^{4}}^{*} f=\varphi+y^{4} \Rightarrow f \in S_{11}$.

95. $j_{x y^{3}}^{*} f=\varphi+x y^{3} \Rightarrow f \in S_{12}$.

Through theorems 96-105, $p>5$.

96. $f=\varphi+\alpha(y)+x \beta(y)+z \gamma(y), j_{x y^{3}}^{*} f=\varphi \Rightarrow$ one of the four possibilities holds:

$$
\begin{array}{rlrl}
j_{y^{5}}^{*} f & \sim_{r} \varphi+y^{5}+b z y^{3}, b^{2} \neq 4 & \mapsto \mathbf{9 7}, \\
& \sim_{r} \varphi+x^{2} y^{2} & \mapsto \mathbf{9 8}, \mathbf{9 9}, \\
& \sim_{r} \varphi+z y^{3} & & \mapsto \mathbf{1 0 0}, \mathbf{1 0 1}, \\
& =\varphi & & \mapsto \mathbf{1 0 2} .
\end{array}
$$

97. $j_{y^{5}}^{*} f=\varphi+y^{5}+b z y^{3}, b^{2} \neq 4 \Rightarrow f \in S_{1,0}$.

98. $j_{y^{5}}^{*} f=\varphi+x^{2} y^{2}$ and $\mu-9<p \Rightarrow f \in S_{1, q}(q:=\mu-14>0)$.

99. $j_{y^{5}}^{*} f=\varphi+x^{2} y^{2}$ and $\mu-9 \geq p \Rightarrow \operatorname{rmod}(f) \geq 3$.

100. $j_{y^{5}}^{*} f=\varphi+z y^{3}$ and $\mu-9<p \Rightarrow f \in S_{1, q}^{\sharp}(q:=\mu-14>0)$.

101. $j_{u^{5}}^{*} f=\varphi+z y^{3}$ and $\mu-9 \geq p \Rightarrow \operatorname{rmod}(f) \geq 3$.

102. $f=\varphi+\alpha(y)+x \beta(y)+z \gamma(y), j_{y^{5}}^{*} f=\varphi \Rightarrow$ one of the three possibilities holds:

$$
\begin{array}{lllll}
j_{x y^{4}}^{*} f & \sim_{r} & \varphi+x y^{4} & \mapsto & \mathbf{1 0 3}, \\
j_{y^{6}}^{*} f & \sim_{r} & \varphi+y^{6} & \mapsto & \mathbf{1 0 4}, \\
j_{y^{6}}^{*} f & = & \varphi & \mapsto & \mathbf{1 0 5} .
\end{array}
$$

103. $j_{x y^{4}}^{*} f=\varphi+x y^{4} \Rightarrow f \in S_{16}$.

104. $j_{y^{6}}^{*} f=\varphi+y^{6} \Rightarrow f \in S_{17}$.

105. $j_{y^{6}}^{*} f=\varphi \Rightarrow \operatorname{rmod}(f) \geq 3$.

106. $f=\varphi+\alpha(y)+x \beta(y)+z \gamma(y), j_{x y^{3}}^{*} f=\varphi$ and $p=5 \Rightarrow \operatorname{rmod}(f) \geq 3$.

\section{Series U}

Through theorems 107-117, $\varphi=x^{3}+x z^{2}, j_{\lambda}^{*}=j_{x^{3}, z^{3}, \lambda},(\lambda$ is a polynomial $)$.

107. $j^{3} f=\varphi \Rightarrow f \sim_{r} \varphi+\alpha(y)+x \beta(y)+z \gamma(y)+x^{2} \delta(y), j^{3}\left(\alpha, x \beta, z \gamma, x^{2} \delta\right)=0 \mapsto 108$. 
108. $f=\varphi+\alpha(y)+x \beta(y)+z \gamma(y)+x^{2} \delta(y), j_{y^{3 k}}^{*} f=\varphi \Rightarrow$ one of the two possibilities holds:

$$
\begin{array}{rlll}
j_{y^{4}}^{*} f & \sim_{r} \varphi+y^{4} & \mapsto & \mathbf{1 0 9}, \\
= & \mapsto & \mathbf{1 1 0} .
\end{array}
$$

109. $j_{y^{4}}^{*} f=\varphi+y^{4} \Rightarrow f \in U_{12}$.

110. $f=\varphi+\alpha(y)+x \beta(y)+z \gamma(y)+x^{2} \delta(y), j_{y^{4}}^{*} f=\varphi \Rightarrow$ one of the three possibilities holds:

$$
\begin{aligned}
& j_{x y^{3}}^{*} f \quad \sim_{r} \quad \varphi+x y^{3}+c z y^{3}, c\left(c^{2}+1\right) \neq 0 \quad \mapsto \mathbf{1 1 1}, \\
& \sim_{r} \varphi+x y^{3} \quad \mapsto \mathbf{1 1 2}, 113, \\
& =\varphi \quad \mapsto \mathbf{1 1 4} .
\end{aligned}
$$

111. $j_{x y^{3}}^{*} f=\varphi+x y^{3}+c z y^{3}, c\left(c^{2}+1\right) \neq 0 \Rightarrow f \in U_{1,0}$.

112. $j_{x y^{3}}^{*} f=\varphi+x y^{3}$ and $\mu-13<p \Rightarrow f \in U_{1, q}(q:=\mu-14 \geq 0)$.

113. $j_{x y^{3}}^{*} f=\varphi+x y^{3}$ and $\mu-13 \geq p \Rightarrow \operatorname{rmod}(f) \geq 3$.

114. $f=\varphi+\alpha(y)+x \beta(y)+z \gamma(y)+x^{2} \delta(y), j_{x y^{3}}^{*} f=\varphi \Rightarrow$ one of the two possibilities holds:

$$
\begin{array}{rll}
j_{y^{5}}^{*} f & \sim_{r} \varphi+y^{5} & \mapsto 115,116 \\
= & \varphi & \mapsto 116,117 .
\end{array}
$$

115. $j_{y^{5}}^{*} f=\varphi+y^{5}$ and $p>5 \Rightarrow f \in U_{16}$.

116. $p=5, f=\varphi+\alpha(y)+x \beta(y)+z \gamma(y)+x^{2} \delta(y)$ and $j_{x y^{3}}^{*} f=\varphi \Rightarrow \operatorname{rmod}(f) \geq 3$.

117. $j_{y^{5}}^{*} f=\varphi \Rightarrow \operatorname{rmod}(f) \geq 3$.

118. $j^{3} f=x^{2} y \Rightarrow f \sim_{r} x^{2} y+\alpha(y, z)+x \beta(z)$ and then $\operatorname{rmod}(f) \geq 3$.

119. $j^{3} f=x^{3} \Rightarrow \operatorname{rmod}(f) \geq 4$.

120. $j^{3} f=0 \Rightarrow \operatorname{rmod}(f) \geq 6$.

\section{Corank $>3$ Singularities}

121. $\operatorname{crk}(\mathrm{f})>3 \Rightarrow \operatorname{rmod}(f) \geq 4$.

\subsection{Singularity determinator in characteristic 2 .}

122. $\mu(f)<\infty \Rightarrow$ one of the three possibilities holds:

$$
\begin{aligned}
\operatorname{crk}(f) & \leq 1 \\
= & \mapsto \mathbf{1 2 3}, \\
\geq 3 & \mapsto \mathbf{1 2 4}, \\
\geq & \mathbf{1 3 6} .
\end{aligned}
$$

123. $\operatorname{crk}(f) \leq 1 \Rightarrow f \in A_{k}(1 \leq k \leq 5)$.

Through theorems , $f \in K[[x, y]]$.

124. $\operatorname{crk}(f)=2 \Rightarrow$ one of the four possibilities holds:

$$
\begin{aligned}
& j^{3} f \quad \sim_{r} a x^{2}+b y^{2}+x^{3}+y^{3} \mapsto \mathbf{1 2 5}, \\
& \sim_{r} a x^{2}+b y^{2}+x^{2} y \quad \mapsto \mathbf{1 2 6}, \\
& \sim_{r} a x^{2}+b y^{2}+x^{3} \quad \mapsto \mathbf{1 3 1}, \\
& =a x^{2}+b y^{2} \quad \mapsto \mathbf{1 3 4}, \mathbf{1 3 5} .
\end{aligned}
$$

125. $j^{3} f=a x^{2}+b y^{2}+x^{3}+y^{3} \Rightarrow f \in D_{4}$.

126. $j^{3} f=a x^{2}+b y^{2}+x^{2} y \Rightarrow$ one of the two possibilities holds:

$$
\begin{aligned}
& j^{4} f \quad \sim_{r} a x^{2}+b y^{2}+x^{2} y+x y^{3} \quad \mapsto \mathbf{1 2 7}, \\
& =a x^{2}+b y^{2}+x^{2} y \quad \mapsto \quad \mathbf{1 2 8}, \mathbf{1 3 0} .
\end{aligned}
$$

127. $j^{4} f=a x^{2}+b y^{2}+x^{2} y+x y^{3} \Rightarrow f \in D_{6}$.

128. $j^{4} f=a x^{2}+b y^{2}+x^{2} y \Rightarrow$ one of the two possibilities holds:

$$
\begin{aligned}
& j^{5} f \quad \sim_{r} a x^{2}+b y^{2}+x^{2} y+x y^{4} \mapsto \mathbf{1 2 9}, \\
& =a x^{2}+b y^{2}+x^{2} y \quad \mapsto \mathbf{1 3 0} .
\end{aligned}
$$

129. $j^{5} f=a x^{2}+b y^{2}+x^{2} y+x y^{4} \Rightarrow$ one of the two possibilities holds:

$$
\begin{aligned}
& j^{5} f \quad \sim_{r} a x^{2}+b y^{2}+x^{2} y+x y^{4}+c x y^{5} \mapsto \mathbf{1 3 0}, \\
& =a x^{2}+b y^{2}+x^{2} y \quad \mapsto \mathbf{1 3 0} .
\end{aligned}
$$

130. $j^{4} f=a x^{2}+b y^{2}+x^{2} y \Rightarrow \operatorname{rmod}(f) \geq 3, \mu(f) \geq 8$.

131. $j^{3} f=a x^{2}+b y^{2}+x^{3} \Rightarrow$ one of the two possibilities holds:

$$
\begin{aligned}
& j^{4} f \sim_{r} a x^{2}+b y^{2}+x^{3}+x y^{3} \mapsto \mathbf{1 3 2} \\
& =a x^{2}+b y^{2}+x^{3} \quad \mapsto \mathbf{1 3 3} .
\end{aligned}
$$


132. $j^{4} f=a x^{2}+b y^{2}+x^{3}+x y^{3} \Rightarrow f \in E_{7}$.

133. $j^{4} f=a x^{2}+b y^{2}+x^{3} \Rightarrow \operatorname{rmod}(f) \geq 3$.

134. $j^{3} f=a x^{2}+b y^{2},(a, b) \neq(0,0) \Rightarrow$ one of the two possibilities holds:

$$
\begin{aligned}
& j^{4} f \quad \sim_{r} a x^{2}+b y^{2}+x^{3} y \quad \mapsto \mathbf{1 3 5}, \\
& =a x^{2}+b y^{2} \quad \mapsto \mathbf{1 3 5} .
\end{aligned}
$$

135. $j^{3} f=a x^{2}+b y^{2} \Rightarrow \operatorname{rmod}(f) \geq 4, \mu(f) \geq 10$.

136. $\operatorname{crk}(f) \geq 3 \Rightarrow \operatorname{rmod}(f) \geq 4, \mu(f) \geq 8$.

\subsection{Singularity determinator in characteristic 3.}

137. $\mu(f)<\infty \Rightarrow$ one of the four possibilities holds:

$$
\begin{aligned}
\operatorname{crk}(f) & \leq 1 \\
=2 & \mapsto \mathbf{1 3 8}, \\
=3 & \mapsto \mathbf{1 3 9 - 1 4 6}, \\
> & \Rightarrow \mapsto \mathbf{1 5 2} .
\end{aligned}
$$

138. $\operatorname{crk}(f) \leq 1 \Rightarrow f \in A_{k}(1 \leq k \leq 8)$.

\section{Corank 2 Singularities}

Through theorems 139-146, $f \in K[[x, y]]$.

139. $j^{2}(f)=0 \Rightarrow$ one of the three possibilities holds:

$$
\begin{aligned}
j^{3} f & \sim_{r} x^{2} y+\epsilon y^{3}, \epsilon \in\{0,1\} & \mapsto \mathbf{1 4 0}, \\
& \sim_{r} x^{3} & \mapsto \mathbf{1 4 5}, \\
& =0 & \mapsto \mathbf{1 4 6} .
\end{aligned}
$$

140. $j^{3}(f)=x^{2} y+\epsilon y^{3}, \epsilon \in\{0,1\} \Rightarrow f \sim_{r} x^{2} y+g(y), j^{2} g=0 \mapsto \mathbf{1 4 1}$.

141. $j^{4} f=x^{2} y+g(y), j^{2} g=0 \Rightarrow$ one of the three possibilities holds:

$$
\begin{aligned}
2<\mu(g)<5 & \mapsto \mathbf{1 4 2}, \\
5<\mu(g)<8 & \mapsto \mathbf{1 4 3}, \\
8<\mu(g) & \mapsto \mathbf{1 4 4} .
\end{aligned}
$$

142. $2<\mu(g)<5 \Rightarrow f \in D_{5}, D_{6}$.

143. $5<\mu(g)<8 \Rightarrow f \in D_{8}, D_{9}$.

144. $8<\mu(g) \Rightarrow \operatorname{rmod}(f) \geq 3, \mu(f) \geq 11$.

145. $j^{3}(f)=x^{3} \Rightarrow \operatorname{rmod}(f) \geq 3, \mu(f) \geq 9$.

146. $j^{3}(f)=0 \Rightarrow \operatorname{rmod}(f) \geq 3, \mu(f) \geq 9$.

\section{Corank 3 Singularities}

Through theorems 147-151, $f \in K[[x, y, z]]$.

147. $j^{2} f(x, y, z)=0 \Rightarrow$ one of the eleven possibilities holds:

$$
\begin{array}{rlll}
j^{3} f & \sim_{r} x^{3}+a x^{2} z+z^{3}+y^{2} z, a \neq 0 & \mapsto \mathbf{1 4 8}, \\
& \sim_{r} x^{3}+a x z^{2}+z^{3}+y^{2} z, a \neq 0 & \mapsto \mathbf{1 4 9}, \\
& \sim_{r} x^{3}+y^{3}+x y z & \mapsto \mathbf{1 5 0}, \\
& \sim_{r} x^{3}+x y z & \mapsto \mathbf{1 5 0}, \\
& \sim_{r} x y z & \mapsto \mathbf{1 5 0}, \\
& \sim_{r} x^{3}+y z^{2} & \mapsto \mathbf{1 5 0}, \\
& \sim_{r} x^{2} z+y z^{2} & \mapsto \mathbf{1 5 0}, \\
& \sim_{r} x^{3}+x z^{2} & \mapsto \mathbf{1 5 0}, \\
& \sim_{r} x^{2} y & \mapsto \mathbf{1 5 0}, \\
& \sim_{r} x^{3} & \mapsto \mathbf{1 5 0}, \\
& =0 & \mapsto \mathbf{1 5 1} .
\end{array}
$$

148. $j^{3}(f)=x^{3}+a x^{2} z+z^{3}+y^{2} z, a \neq 0 \Rightarrow \operatorname{rmod}(f) \geq 4, \mu(f) \geq 11$.

149. $j^{3}(f)=x^{3}+a x z^{2}+z^{3}+y^{2} z, a \neq 0 \Rightarrow \operatorname{rmod}(f) \geq 4, \mu(f) \geq 11$.

150. $j^{3}(f)$ is degenerate $\Rightarrow \operatorname{rmod}(f) \geq 4, \mu(f) \geq 11$.

151. $j^{3} f=0 \Rightarrow \operatorname{rmod}(f) \geq 6$.

\section{Corank $>3$ Singularities}

152. $\operatorname{crk}(\mathrm{f})>3 \Rightarrow \operatorname{rmod}(f) \geq 4$. 


\section{Proof OF the MAIN RESUlts}

We first use the splitting lemma to reduce the number of variables. Namely, if $f \in \mathfrak{m}^{2} \subset K[[\mathbf{x}]]$ has corank, $\operatorname{crk}(f)=k \geq 0$, then

$$
f \sim_{r} g\left(x_{1}, \ldots, x_{k}\right)+Q\left(x_{k+1}, \ldots, x_{n}\right)
$$

with $g \in \mathfrak{m}^{3}$ and $Q$ is a nondegenerate quadratic singularity (cf. [14, Lemma 3.9, 3.12]). One has moreover that $\operatorname{rmod}(f)$ in $K[[\mathbf{x}]]$ is equal to $\operatorname{rmod}(g)$ in $K\left[\left[x_{1}, \ldots, x_{k}\right]\right]$, cf. [14, Lemma 3.11, 3.13].

Theorems 1, 92, 122, 137 and 141 are obvious. Theorems 9, 17, 20, 25, 39, 40, 44, 54, 57, 66, $\mathbf{8 2}, \mathbf{9 3}, \mathbf{1 0 2}, \mathbf{1 0 8}, \mathbf{1 1 4}$ are proved by the Newton method [20] of a moving ruler (line, plane). This method reduces the proof to the counting of the integer points in triangles resp. polyhedrones on the exponent plane (resp. in the space).

Theorems concerning the geometrical classification problems: The proofs of theorems $\mathbf{4}, \mathbf{1 3}, \mathbf{2 6}, \mathbf{3 1}$, $49,60,72,74,96,110,124,139,147$ can be reduced to the classifications of orbits of the actions of some quasihomogenous diffeomorphism groups on the spaces of quasihomenous polynomials, see Section 5.1 for a proof of Theorem 147.

Theorems on normal forms: Theorems 2, 123, 138 follow from [22, Thm 2.11]. The proofs of theorems $5,6,7,10,11,12,14,15,18,21,22,27,28,30,32,33,37,38,41,42,43,45,46$, $50,51,55,56,58,61,62,64,67,68,72,75,76,77,79,80,81,83,84,85,88,89,94,95,97$, $98,100,103,104,107,109,111,112,115,125,127,132,142,143$ are based on the techniques introduced in [2] and generalized in [9], see Section 5.2 for a proof of Theorem 14.

Theorems on low bound of modality: Theorems 121, 151, 152 are consequences of [14, Prop. 2.18]. Theorems 3, 8, 16, 19, 23, 24, 25, 29, 34, 36, 47, 48, 52, 53, 59, 63, 65, 69, 70, 72, 73, 78, 79(ii), 80(iii), 86, 90, 99, 105, 106, 113, 116-120, 130, 133, 135, 136, 144, 145, 146, 148, 149, 150 are proved by using the theory in [14] (21]), see Section 5.3 for a proof of $\mathbf{2 5}$ and $\mathbf{7 0 .}$

Theorems on adjacencies: Theorem 3.5 is proved inductively by applying Theorems $\mathbf{1}, \mathbf{2}, \mathbf{4 - 7}, \mathbf{8}-$ $15,17,18,20,21,22,26,27,28,30-33,35,37-46,49-51,54-58,60-62,64,66-68,74-77$, 79-85, 87-89, 91-98, 100-104, 107-112, 114-117, 122-127, 131, 132, 137-143.

Classification of unimodal and bimodal singularities (Theorems 3.1 3.4): Applying Theorems 1-153 and the spliting lemma (cf. [14]) we obtain the list of families of singularities in Tables 1-11. The modularity of these families follows from simple caculations. To prove these singularities are unimodal resp. bimodal we use the theory of modality in [14. See Section 5.4 for a proof that $E_{12}$ with $p>7$, is a class of unimodal singularities.

Smoothness of $\mu$-constant stratum and proper modality (Corollaries 3.8, 3.9): are proved by using adjacency diagrams (Theorem 3.5). See Section 5.4 for a proof that if $f$ is of type $T_{q, r, s}$ as in Table 8 . then $\mu$-constant stratum $\Delta_{\mu}$ of $f$ is isomorphic to $\mathbb{A}^{2}$. This also show that $\operatorname{rmod}(f)=\operatorname{pmod}(f)=2$.

5.1. Proof of Theorem 147. The theorem is obtained by combining the following lemmas 5.15 .2 , 5.3. Let $0 \neq f \in K[x, y, z]$, with $\operatorname{char}(K)=3$, be a homogeneous polynomial of degree 3 .

Lemma 5.1. If $f$ is nonsingular, then $f$ is right equivalent to one of the following forms $(a \neq 0)$

$$
x^{3}+a x^{2} z+z^{3}+y^{2} z, x^{3}+a x z^{2}+z^{3}+y^{2} z .
$$

Proof. cf. [18, Chap. II, Prop.1.2]

Lemma 5.2. If $f$ is singular in $\mathbb{P}_{K}^{2}$ and irreducible, then it is right equivalent to one of the following forms

$$
x^{3}+y^{3}+x y z, x^{3}+y^{2} z
$$

Proof. Let $C$ be the curve in $\mathbb{P}^{2}$ defined by $f$. Take $P \in \operatorname{Sing}(C)$ and $P \neq Q \in C$. Let $L$ be the line in $\mathbb{P}^{2}$ connecting $P, Q$. Applying Bézout theorem we obtain that

$$
3=\operatorname{deg}(C) \cdot \operatorname{deg}(L) \geq \operatorname{mt}_{P}(C)+\operatorname{mt}_{Q}(C) .
$$


Hence $\operatorname{mt}_{P}(C)=2$ and $\operatorname{mt}_{Q}(C)=1$. We may assume $P=(0: 0: 1)$ and set $g(x, y):=f(x, y, 1)$. Then $\operatorname{mt}(g)=2$ since $\operatorname{mt}_{P}(C)=2$. It yields that $g$ is right equivalent to one of the following forms

$$
x y+h(x, y), y^{2}+h(x, y)
$$

with $h(x, y)$ is a homogeneous polynomial of degree 3 . That is, $f$ is right equivalent to either

$$
x y z+h(x, y) \text { or } y^{2} z+h(x, y) \text {. }
$$

It hence follows by simple calculations that $f$ is right equivalent to one of the two forms

$$
x^{3}+y^{3}+x y z, x^{3}+y^{2} z .
$$

Lemma 5.3. If $f$ is reducible, then it is right equivalent to one of the following forms

$$
x^{3}, x^{2} y, x^{2} z+y z^{2}, x^{3}+x y z, x^{3}+x z^{2}, x y z .
$$

Proof. Let $f=g_{1} \cdot g_{2}$ with $\operatorname{mt}\left(g_{1}\right)=1, \operatorname{mt}\left(g_{2}\right)=2$. By the splitting lemma (cf. [14])

$$
g_{2} \sim_{r} a x^{2}+b y z
$$

with $a, b \in\{0,1\}$. That is $f \sim_{r} g_{1} \cdot\left(a x^{2}+b y z\right)$. Consider the following cases:

- $a=1, b=0$ : Then $f$ is right equivalent to $x^{3}$ or $x^{2} y$.

- $a=1, b=1$ : Then $f \sim_{r} g_{1} \cdot\left(x^{2}+y z\right)$. Without loss of generality we may assume moreover that

$$
\{(0: 1: 0)\} \in\left\{g_{1}=0\right\} \cap\left\{x^{2}+y z=0\right\},
$$

i.e. $g_{1}$ has the form $g_{1}=\alpha x+\beta z$.

- If $\alpha=0$, then $f \sim_{r} z\left(x^{2}+y z\right)$,

- if $\alpha \neq 0$, then $f \sim x\left(x^{2}+y z\right)$.

- $a=0, b=1$ : Then $f \sim_{r} g_{1} \cdot y z$. It yields that $f$ is right equivalent to one of the forms: $y^{2} z, x y z,(y+$ $z) y z$, or, one of the forms: $x^{2} y, x y z, x^{3}+x z^{2}$.

5.2. Proof of Theorem 14. Let $f \in K[[x, y]]$ with $p=\operatorname{char}(K)=5$ and $j_{x^{3}, y^{6}} f=x^{3}+b x^{2} y^{2}+y^{6}+$ $a y^{5}$. We will show that $f$ is right equivalent to $f_{0}:=x^{3}+b x^{2} y^{2}+y^{6}+a y^{5}$, i.e. $f$ is of type $J_{2,0}$.

In fact, put $g:=f-a y^{5}$, then $j_{x^{3}, y^{6}} g=x^{3}+b x^{2} y^{2}+y^{6}$. Applying [8, Thm. 4.4] we obtain that $g \sim_{r} x^{3}+b x^{2} y^{2}+y^{6}$. We can see moreover that there exists a coordinate change of the form

$$
x \mapsto x+\varphi_{1}(x, y), y \mapsto y+\varphi_{2}(x, y)
$$

with $\operatorname{mt}\left(\varphi_{i}\right) \geq 2$ such that

$$
g\left(x+\varphi_{1}, y+\varphi_{2}\right)=x^{3}+b x^{2} y^{2}+y^{6} .
$$

It yields

$$
f_{1}:=f\left(x+\varphi_{1}, y+\varphi_{2}\right)=x^{3}+b x^{2} y^{2}+y^{6}+a\left(y+\varphi_{2}\right)^{5}=x^{3}+b x^{2} y^{2}+y^{6}+a y^{5}+a \varphi_{2}^{5} .
$$

It is easy to see that $\mathfrak{m}^{7} \subset \mathfrak{m}^{2} \cdot j\left(f_{1}\right)$. By [9, Thm. 2.1], $f_{1}$ is right 9-determined and hence $f_{1} \sim_{r} f_{0}$ since $\operatorname{mt}\left(\varphi_{2}^{5}\right) \geq 10$. This completes the proof.

5.3. Proof of theorems on lower bound of modality. For the proof of these theorems we need the following lemma which is deduced from Corollaries A.4, A.9, A.10 of [14 (see 21, Prop. 3.2.4, Cor. 3.3.4 and Cor. 3.3.6] for more details).

Lemma 5.4. Let the algebraic groups $G$ resp. $G^{\prime}$ act on the varieties $X$ resp. $X^{\prime}$. Let $h: Y \rightarrow X a$ morphism of varieties and let $h^{\prime}: Y \rightarrow X^{\prime}$ an open morphism such that

$$
h^{-1}(G \cdot h(y)) \subset h^{\prime-1}\left(G^{\prime} \cdot h^{\prime}(y)\right), \forall y \in Y .
$$

Then for all $y \in Y$ we have

$$
G-\bmod (h(y)) \geq G^{\prime}-\bmod \left(h^{\prime}(y)\right) \geq \operatorname{dim} X^{\prime}-\operatorname{dim} G^{\prime} .
$$

Lemma 5.5. Let $f \in K[[x, y]]$ with $\operatorname{char}(K)>3$. Then $\operatorname{rmod}(f) \geq 2+l$ with $l \geq 0$, if either (i) $f \in\left\langle x, y^{3+l}\right\rangle^{3}$; or 
(ii) $f \in\left\langle x^{2}, y^{3+l}\right\rangle^{2}$.

Proof. We prove only for (i) since the proof for (ii) is similar. Let $k$ be sufficiently large for $f$, i.e. $\operatorname{rmod}(f)=\mathcal{R}_{k}-\bmod (f)$. We denote

$$
\begin{gathered}
\Delta:=\{(3 ; s),(2 ; 3+s),(1 ; 6+s),(0 ; 9+s) \mid 0 \leq s \leq l+1\} \subset \mathbb{N}^{2}, \\
\left.\Delta_{1}:=\{(1 ; s),(0 ; 3+l+s) \mid 0 \leq s \leq l+1\} \text { and } \Delta_{2}:=\{(0 ; 1+s) \mid 0 \leq s \leq l+1\}\right\} \subset \mathbb{N}^{2}
\end{gathered}
$$

and define

$$
\begin{gathered}
X:=\left\{\sum_{(i, j) \in \Delta} a_{i, j} x^{i} y^{j} \in K[[x, y]] \mid a_{i, j} \in K\right\} \cong \mathbb{A}^{4(l+2)}, \\
G:=X_{1} \times X_{2} \cong \mathbb{A}^{3(l+2)},
\end{gathered}
$$

where

$$
\begin{aligned}
& X_{1}:=\left\{\sum_{(i, j) \in \Delta_{1}} a_{i, j} x^{i} y^{j} \in K[[x, y]] \mid a_{i, j} \in K, a_{10} \neq 0\right\}, \\
& X_{2}:=\left\{\sum_{(i, j) \in \Delta_{2}} b_{i, j} x^{i} y^{j} \in K[[x, y]] \mid b_{i, j} \in K, b_{01} \neq 0\right\} .
\end{aligned}
$$

Using the projections

$$
\begin{aligned}
\pi_{1}: J_{k} \rightarrow X_{1}, \sum_{(i, j)} a_{i, j} x^{i} y^{j} \mapsto \sum_{(i, j) \in \Delta_{1}} a_{i, j} x^{i} y^{j}, \\
\pi_{2}: J_{k} \rightarrow X_{2}, \sum_{(i, j)} a_{i, j} x^{i} y^{j} \mapsto \sum_{(i, j) \in \Delta_{2}} a_{i, j} x^{i} y^{j}, \\
\pi: J_{k} \rightarrow X, \sum_{(i, j)} a_{i, j} x^{i} y^{j} \mapsto \sum_{(i, j) \in \Delta} a_{i, j} x^{i} y^{j}
\end{aligned}
$$

and

$$
\begin{aligned}
\bar{\pi}: \mathcal{R}_{k} & \rightarrow G=X_{1} \times X_{2} \\
\Phi=\left(\Phi_{1}, \Phi_{2}\right) & \mapsto\left(\pi_{1}\left(\Phi_{1}\right), \pi_{2}\left(\Phi_{2}\right)\right)
\end{aligned}
$$

we may define a multiplication on $G$, resp. an action map of $G$ on $X$ as follows

$$
\begin{aligned}
\bullet: G \times G & \rightarrow G \\
\left(\phi, \phi^{\prime}\right) & \mapsto \bar{\pi}\left(\phi \circ \phi^{\prime}\right),
\end{aligned}
$$

resp.

$$
\begin{aligned}
G \times X & \rightarrow X \\
(\phi, g) & \mapsto \pi(\phi(g)) .
\end{aligned}
$$

By a simple calculation we can verify that the morphisms $\iota: Y:=\left\langle x, y^{3+l}\right\rangle^{3} / \mathfrak{m}^{k+1} \hookrightarrow J_{k}$ and $\pi: Y \rightarrow X$ satisfy

$$
\iota^{-1}\left(\mathcal{R}_{k} \cdot \iota(g)\right) \subset \pi^{-1}(G \cdot \pi(g)), \forall g \in Y .
$$

Hence applying Lemma 5.4 we obtain that

$$
\operatorname{rmod}(f)=\mathcal{R}_{k}-\bmod (\iota(f)) \geq \operatorname{dim} X-\operatorname{dim} G=2+l .
$$


5.4. Computing the modality of $E_{12}$. We shall show that $E_{12}$ is a class of unimodal singularities. To compute the modality of a singularity we use the general argument in [14, in particular, the following lemma.

Lemma 5.6. Assume that $f \in K[[\mathbf{x}]]$ deforms only into finitely many families $h_{t}^{(i)}(\mathbf{x})$ over varieties $T^{(i)}, i \in I$. Then

$$
\operatorname{rmod}(f) \leq \max _{i \in I} \operatorname{dim} T^{(i)}
$$

Assume further that the families $h_{t}^{(i)}(\mathbf{x})$ are all modular. Then

$$
\operatorname{rmod}(f)=\max _{i \in I} \operatorname{dim} T^{(i)} \text {. }
$$

Proof. cf. [14, Prop. 2.15.

Proof for $E_{12}$. Assume that $f=x^{3}+y^{7}+a x y^{5} \in K[[x, y]]$ with $p=\operatorname{char}(K)>7$ and $a \in K$, is of type $E_{12}$. We will show that

$$
\operatorname{rmod}(f)=1 \text {. }
$$

In fact, by Theorem 3.5 (or, Theorems 1-9, 26, 27, 28), $f$ deforms only into the following modular families

$$
E_{12}, A_{k}(k \leq 6), D_{k}(k \leq 8), E_{6}, E_{7}, E_{8}, J_{2,0}, J_{2,1} .
$$

Hence it follows from Lemma 5.6 that $f$ is right unimodal singularities.

5.5. Smoothness of $\mu$-constant stratum and proper modality. Let $f=x^{q}+y^{r}+z^{s}+a x y z+b z^{p}$ be of type $T_{q, r, s}$ with $3 \leq q \leq r<p \leq s<2 p$ as in Table 8 . Then

$$
\mathfrak{m} / \mathfrak{m} \cdot j(f)=\left\{x, \ldots, x^{q-1}, y, \ldots, y^{r-1}, z, \ldots, z^{s-1}, x y, y z, z x, x y z\right\}
$$

and the semiuniversal unfolding $f_{\lambda}$ of $f$ over $\mathbb{A}^{q+r+s+1}, 0$ is given by

$$
f_{\lambda}=f+\sum_{i=1}^{q-1} a_{i} x^{i}+\sum_{j=1}^{r-1} b_{j} y^{j}+\sum_{l=1}^{s-1} a_{l} z^{l}+d_{1} x y+d_{2} y z+d_{3} z x+d_{4} x y z
$$

with $\lambda=\left(a_{1}, \ldots, a_{q-1}, b_{1} \ldots, b_{r-1}, c_{1} \ldots, c_{s-1}, d_{1}, d_{2}, d_{3}, d_{4}\right)$ the coordinate of $\lambda \in \mathbb{A}^{q+r+s+1}$.

Consider the $\mu$-constant stratum $\Delta_{\mu}$ of $f$, and assume that $\lambda \in \Delta_{\mu}$. It follows Theorem 3.5 that

$$
a_{1}=\cdots=a_{q-1}=b_{1}=\cdots=b_{r-1}=c_{1}=\cdots=c_{p-1}=c_{p+1}=\cdots=c_{s-1}=d_{1}=d_{2}=d_{3}=0 .
$$

Moreover Theorems $\mathbf{7 6}, \mathbf{7 7}, \mathbf{7 9}, \mathbf{8 0}$ yield that, $c_{p}, d_{4}$ can be arbitrary, and hence $\Delta_{\mu} \cong \mathbb{A}^{2}$. This implies that $\operatorname{rmod}(f)=\operatorname{pmod}(f)=2$.

Acknowledgement. The author would like to thank Gert-Martin Greuel for the numerous fruitful discussions and for many useful comments on this text.

\section{REFERENCES}

[1] V. I. Arnol'd, S. M. Gusein-Zade, and A.N. Varchenko, Singularities of differentiable maps, Vol I. Birkhäuser (1985).

[2] V. I. Arnol'd, Normal forms for functions near degenerate critical points, the Weyl groups of $A_{k}, D_{k}, E_{k}$ and Lagrangian singularities, Funct. Anal. Appl. 6 (1972), 254-272.

[3] V. I. Arnol'd, Classification of unimodal critical points of functions, Funct. Anal. Appl. 7 (1973), $230-231$.

[4] V. I. Arnol'd, Normal forms of functions in the neighbourhoods of degenerate critical points, Russian Math. surveys 29 (1974), no. 2, 11-49.

[5] V. I. Arnol'd, Classification of bimodal critical points of functions, Funct. Anal. Appl. 9 (1975), no. 1, $43-44$.

[6] V. I. Arnol'd, Local normal form of functions, Invent. Math. 35 (1976), 87-109.

[7] M. Artin, Coverings of the rational double points in characteristic p, in: Complex Analysis and Algebraic Geometry, ed. W. L. Baily, jr. and T. Shioda, Iwanami Shoten, Publ., Cambridge Univ. Press, 1977. 
[8] Y. Boubakri, G.-M. Greuel, and T. Markwig, Normal forms of hypersurface singularities in positive characteristic, Mosc. Math. J. 11(2011), no. 4, 657-683.

[9] Y. Boubakri, G.-M. Greuel, and T. Markwig, Invariants of hypersurface singularities in positive characteristic, Rev. Mat. Complut. 25(2012), no. 1, 61-85.

[10] A. M. Gabrielov, Bifurcations, Dynkin diagrams and the modality of isolated singularities, Funktsional. Anal. i Prilozhen. 8:2, (1974), 7-12.

(Engl. translation: Funct. Anal. Appl. 8 (1974), 94-98.)

[11] M. Giusti, Classification des Singularitiés isolées d'intersectios compleètes simples, C. R. Math. Acad. Sci. Paris 284(1977), no. 3, A167-A170.

[12] G.-M. Greuel, H. Kröning, Simple singularities in positive characteristic, Math. Z. 203 (1990), 339-354.

[13] G.-M. Greuel, C. Lossen and E. Shustin, Introduction to Singularities and deformations, Math. Monographs, Springer-Verlag (2006).

[14] G.-M. Greuel, H. D. Nguyen, Right simple singularities in positive characteristic, J. Reine Angew. Math. 712 (2016), 81-106.

[15] A. Kas, M. Schlessinger, On the versal deformation of a complex space with an isolated singularity, Math. Ann. 196 (1972), 23-29.

[16] V.S. Kulikov, Degenerate elliptic curves and resolution of uni- and bimodal singularities, Funct. Anal. Appl. 9 (1975), no.1, 69-70.

[17] I. Luengo, The $\mu$-constant stratum is not smooth, Invent. Math., 90 (1987), 139-152.

[18] J. S. Milne, Elliptic Curves, BookSurge Publ., 2006.

[19] J. Milnor, Singular points of complex hypersurfaces, Princeton Univ. Press (1968).

[20] I. Newton, La méthode des fluxions, et des suites infinies, A Paris : Chez Debure l'aîné (1740).

[21] H. D. Nguyen, Classification of singularities in positive characteristic, Ph.D. thesis, TU Kaiserslautern (2013).

[22] H. D. Nguyen, The right classification of univariate singularities in positive characteristic, J. Singul. 10 (2014), $235-249$.

[23] M. Suzuki, E. Yoshinaga, Normal forms of non-degenerate quasihomogeneous functions with inner modality $\leq 4$, Invent. Math. 55 (1979), 185-206.

[24] C. T. C. Wall, Classification of unimodal isolated singularities of complete intersections, pp 625-640 in Proc. Symp. in Pure Math. 40ii (Singularities) (ed. P. Orlik) Amer. Math. Soc., 1983.

Hanoi Institute of Mathematics

18 Hoang Quoc Viet, Hanoi, Vietnam.

E-mail address: nhduc82@gmail.com

Basque Center for Applied Mathematics,

Alameda de Mazarredo 14, 48009 Bilbao, Bizkaia, Spain.

E-mail address: hnguyen@bcamath.org 